\title{
A NOVEL HIGH QUANTUM EFFICIENCY MV X-RAY DETECTOR FOR IMAGE-GUIDED RADIOTHERAPY
}

by

\author{
Jian Liu \\ Doctor of Philosophy, The University of Western Ontario, 2009 \\ Master of Science, Wuhan University, 2004 \\ Bachelor of Science, Wuhan University, 2001
}

A thesis

presented to Ryerson University

in partial fulfillment of the

requirements for the degree of

Master of Science

in the program of

Biomedical Physics

Toronto, Ontario, Canada, 2018

(C)Jian Liu, 2018 


\section{AUTHOR'S DECLARATION}

I hereby declare that I am the sole author of this thesis. This is a true copy of the thesis, including any required final revisions, as accepted by my examiners.

I authorize Ryerson University to lend this thesis to other institutions or individuals for the purpose of scholarly research.

I further authorize Ryerson University to reproduce this thesis by photocopying or by other means, in total or in part, at the request of other institutions or individuals for the purpose of scholarly research.

I understand that my dissertation may be made electronically available to the public.

Jian Liu 
A novel high quantum efficiency MV x-ray detector for image-guided radiotherapy

Master of Science, 2018

Jian Liu

Biomedical Physics

Ryerson University

\begin{abstract}
To develop a new MV x-ray detector with a high quantum efficiency and an adequate spatial resolution for image-guided radiotherapy, scintillating fibers with a diameter of $1 \mathrm{~mm}$ were embedded in lead to form a honeycomb pattern with a thickness of $2 \mathrm{~cm}$. The properties of the detector were measured using a $6 \mathrm{MV}$ beam on a Linac machine. The prototype detector has a quantum efficient of $35 \%$, about an order of magnitude higher than that of current detectors used in the clinic. The spatial resolution of the prototype is comparable to that of video-based electronic portal imaging systems. The prototype detector can also suppress scattered signals which will help to improve the signal to noise ratio of the image. This work indicates that using scintillating fibers to generate and guide imaging signals, it is possible to increase the quantum efficiency and maintain an adequate spatial resolution for MV x-ray imaging.
\end{abstract}




\section{Acknowledgements}

I would like to thank my supervisor, Dr. Geordi Pang, for his support to my research and course work. His extensive knowledge and guidance have made my research enjoyable and fruitful. I am grateful for having had the opportunity to complete a master's degree in Biomedical Physics with CAMPEP accreditation, which will certainly lead me to a new and exciting career.

I would like to thank my co-supervisor, Dr. Yuan $\mathrm{Xu}$, for his support and help with my study at Ryerson University. I benefited greatly from his insightful opinion and constructive suggestions on my research.

I also would like to thank Dr. James Gräfe and Dr. Arman Sarfehnia for their helpful discussions in my supervisory committee meetings.

My sincere thanks also goes to Dr. Aram Teymurazyan for providing the prototype detector.

I am most appreciative of the excellent technical assistance of several individuals which has benefited my research: specifically Philip Au at the Odette Cancer Centre, Sunnybrook Health Sciences Centre; Kevin Liu, Dr. Graham Ferrier and Graham Pearson at Ryerson University.

I gratefully acknowledge the funding sources, NSERC (Natural Sciences and Engineering Research Council of Canada) and OGS (Ontario Graduate Scholarship), which made this work possible.

Lastly, I would like to thank my wife, Lihua Li, for supporting me to pursue my master's degree and a career in Biomedical Physics. 


\section{Contents}

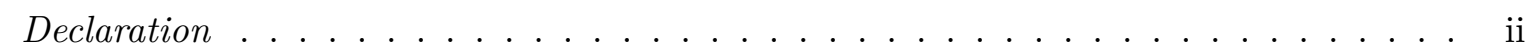

Abstract . . . . . . . . . . . . . . . . . . . . . iii

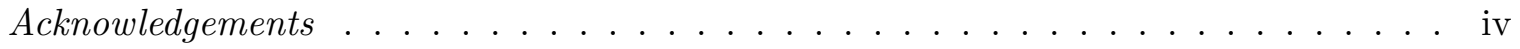

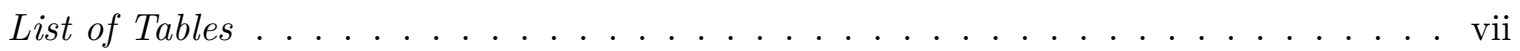

List of Figures . . . . . . . . . . . . . . . . . . . . . viii

List of Appendices . . . . . . . . . . . . . . . . . . . . . x

List of Symbols . . . . . . . . . . . . . . . . . . . . xi

\begin{tabular}{lll}
\hline & Introduction & 1
\end{tabular}

1.1 Importance of MV x-ray imaging $\ldots \ldots \ldots \ldots \ldots \ldots$

1.2 Principle of MV x-ray imaging . . . . . . . . . . . . . . . . . 3

1.3 Important physical processes in x-ray detection . . . . . . . . . . . . . . . . . 6

1.4 Parameters that characterize the quality of x-ray images and the performance of imaging systems $\ldots \ldots \ldots \ldots \ldots \ldots \ldots$

1.4 .1 Contrast $\ldots \ldots \ldots \ldots \ldots \ldots \ldots$

1.4 .2 Signal to noise ratio $(\mathrm{SNR}) \ldots \ldots \ldots \ldots \ldots \ldots$

1.4 .3 Spatial resolution . . . . . . . . . . . . . . . . . . 11

\begin{tabular}{|l|l|l|l|l|}
\hline 1.4 .4 & Quantum efficiency (QE) & and & detective quantum efficiency (DQE) . . . . 12
\end{tabular}

1.5 Current challenges with MV x-ray imaging $\ldots \ldots \ldots \ldots \ldots$

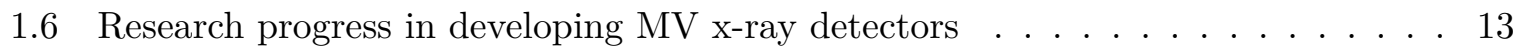

1.7 Objective of this project $\ldots \ldots \ldots \ldots \ldots \ldots \ldots$

\begin{tabular}{|lll}
2 & Materials and methods & 17
\end{tabular}

2.1 Scintillating fiber emission spectrum . . . . . . . . . . . . . . . . . . . . 19

2.2 Signal attenuation due to the optical dual switch and optical fiber . . . . . . . . 19

2.3 Linearity . . . . . . . . . . . . . . . . . . . . . . . . . . . . . 19

2.4 Quantum efficiency . . . . . . . . . . . . . . . . . . . . . . . 20

2.5 Spatial resolution . . . . . . . . . . . . . . . . . . . . . . 20 


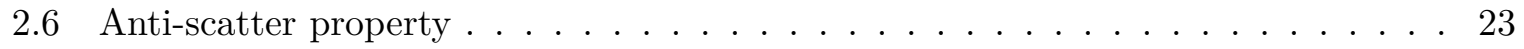

2.7 Sensitivity of the detector $\ldots \ldots \ldots \ldots \ldots \ldots \ldots$

$\begin{array}{lll}3 & \text { Results and discussion } & 29\end{array}$

3.1 Scintillating fiber emission spectrum $\ldots \ldots \ldots \ldots$

3.2 Signal attenuation due to the optical dual switch and optical fiber . . . . . . . . 30

3.3 Linearity . . . . . . . . . . . . . . . . . . . . . . . . . . 30

3.4 Quantum efficiency . . . . . . . . . . . . . . . . . . . . 34

3.5 Spatial resolution . . . . . . . . . . . . . . . . . . . . . . 34

3.6 Anti-scatter property $\ldots \ldots \ldots \ldots \ldots \ldots \ldots$

3.7 Sensitivity of the detector $\ldots \ldots \ldots \ldots \ldots \ldots$

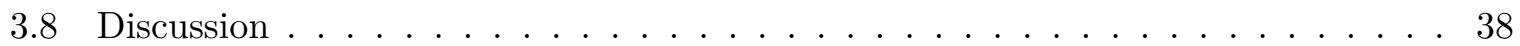

\begin{tabular}{|ll}
\hline Conclusion and future work & 41
\end{tabular}

4.1 Conclusion $\ldots \ldots \ldots \ldots \ldots \ldots \ldots$

4.2 Future work . . . . . . . . . . . . . . . . . . . . . . . . . . . 41

\begin{tabular}{ll}
\hline References & 45
\end{tabular}

\begin{tabular}{ll}
\hline Acronyms & 51
\end{tabular} 


\section{List of Tables}

2.1 Material properties of the Kurary SCSF-78MJ scintillating fiber. . . . . . . . . . 17

$\begin{array}{|ll|}3.1 & \text { Ratios }\left(R_{0}\right) \text { of the primary signals in the presence of the phantom to those } \\ \text { without the phantom at different air gaps. }\end{array}$ 


\section{List of Figures}

1.1 A Linac with a kV imaging system attached perpendicular to the treatment beam in the treatment room. . . . . . . . . . . . . . . . . . . 2

1.2 Illustration of the acquisition of a portal image. . . . . . . . . . . . . . 4

$1.3 \quad$ Illustration of working principle of a direct converter flat-panel detector. Adapted from Ref. $\mid 10]$. . . . . . . . . . . . . . . . . . . . . . . 4 4

1.4 Illustration of working principle of an indirect converter flat-panel detector. Adapted from Ref. [10] . . . . . . . . . . . . . . . 5 5

1.5 X-ray spectrum generated by a treatment planning system at 6 MV (Pinnacle3,

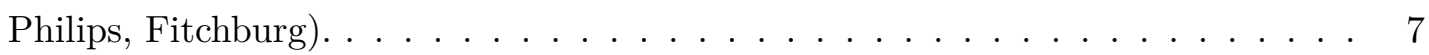

$1.6 \quad$ Illustration of photon fluence attenuated by detecting material. . . . . . . . . . 8

1.7 Mass attenuation coefficients due to photoelectric effect, Compton effect and pair production for $\mathrm{x}$ rays in copper. Solid line is the sum of three mass attenuation coefficients. Data source: Ref. $|14| . \ldots \ldots \ldots$. . . . . . . . . . 9

1.8 Schematic representation of the portal imaging process. $\phi$ is x-ray fluence. Adapted from Ref. $[15]$. . . . . . . . . . . . . . . . . 10

1.9 Illustration of the signal production and conduction in the proposed detector. . . 16

2.1 Diagram of the prototype detector structure $\ldots \ldots \ldots \ldots \ldots$

2.2 The prototype detector placed on top of the "Ryerson University" logo. . . . . . 18

2.3 Diagram showing the measurement of signal attenuated by the optical switch and the optical fiber. . . . . . . . . . . . . . . 20

2.4 Experimental setup for linearity measurement of the detector. . . . . . . . . . . . 21

2.5 Experimental setup for line spread function measurement. . . . . . . . . . . . . . 22

2.6 Ionization chamber reading as a function of rotation stage position. The peak location indicates that the slit object in Fig. 2.5 is aligned to the incident x-ray

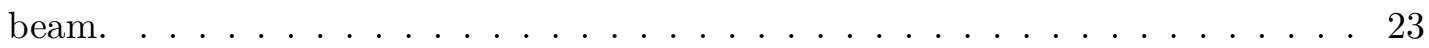

2.7 Experimental setup for measuring the anti-scatter properties of the detector. . . 24 
2.8 Experimental setup for measuring signal from the fiber segment outside the de-

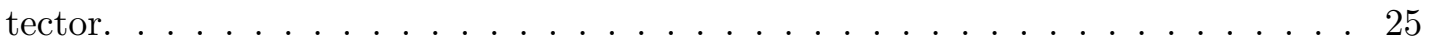

2.9 Detector signal as a function of field sizes for an air gap of $15 \mathrm{~cm}$. Dashed line is the extrapolation to field size zero. . . . . . . . . . . . . . . . 26

3.1 Scintillating fiber emission spectrum excited by a 6 MV beam. Red line is the average of 78 measurements (black lines). . . . . . . . . . . . . . . . . . . 30

3.2 Cathode radiant sensitivity and quantum efficiency of the photomultiplier tube (PMT) used in this work as a function of wavelength (Source: manufacturer product data sheet). . . . . . . . . . . . . . . . . 31

$3.3 \quad$ Scintillating fiber emission spectra excited by a $6 \mathrm{MV}$ beam at different gantry angles. . . . . . . . . . . . . . . . . . . . . . 32

$3.4 \quad$ Scintillating fiber emission spectra excited by $\mathrm{kV}$ and MV beams. . . . . . . . . . 32

3.5 Lamp emission spectrum measured using the setup shown in Fig. 2.3 . . . . . . . 33

$3.6 \quad$ Transmission ratio of the light intensity through the optical switch and the optical

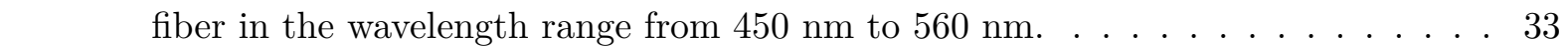

3.7 Detector signal measured by the photomultiplier tube as a function of incident

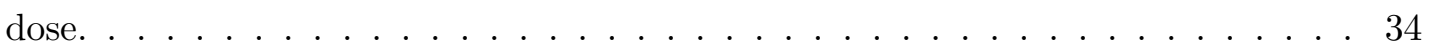

3.8 Line spread function of the detector in the $\mathrm{x}$ and $\mathrm{y}$ directions. . . . . . . . . . . 35

3.9 Modulation transfer function of the detector in the $\mathrm{x}$ and $\mathrm{y}$ directions. . . . . . . 36

3.10 Detector signals due to the primary beam with and without phantom as a function of source to detector distance. . . . . . . . . . . . . . . . . . . 36

3.11 Scatter to primary ratios as a function of the field size at different air gaps for the prototype detector and ionization chamber detector. . . . . . . . . . . . 37

3.12 PMT reading distribution for detector sensitivity measurement. . . . . . . . . . . 38

3.13 Diagram showing the optical photons that are excited in the scintillating fiber and able to exit the fiber. . . . . . . . . . . . . . . . . . . . 40 


\section{List of Appendices}

1 Data acquired in measurements of the detector sensitivity 


\section{List of Symbols}

$\delta \quad$ average $\mathrm{PMT}$ gain per stage

$\eta \quad$ average optical quantum efficiency of the PMT

$\eta_{d} \quad$ detector efficiency

$\gamma \quad$ light transmission coefficient through the optical dual switch and the optical fiber

$\mu \quad$ x-ray linear attenuation coefficient

$\bar{d} \quad$ mean detector signal

$\bar{q} \quad$ mean incident x-ray fluence

$\phi_{i} \quad$ incident fluence

$\phi_{p} \quad$ primary photon fluence

$\phi_{s} \quad$ scattered photon fluence

$\rho \quad$ density

$\sigma_{p h} \quad$ root-mean-square noise of the signal per pixel

$\xi \quad$ light transmission coefficient at the interface between the optical fiber and the PMT

$A \quad$ area

e electron charge

$G \quad$ PMT gain

$N_{p h} \quad$ number of scintillating photons detected per pixel of the detector

$P \quad$ primary signal in the presence of the phantom 
$P^{\prime} \quad$ primary signal without the presence of the phantom

$Q \quad$ charge

$S \quad$ scatter signal in the presence of the phantom

$S^{\prime} \quad$ scatter signal without the presence of the phantom

$T \quad$ total signal in the presence of the phantom

$T^{\prime} \quad$ total signal without the presence of the phantom

$T_{\mathrm{x}} \quad$ fraction of transmitted $\mathrm{x}$ rays

$\sigma_{P M T}^{2} \quad$ variance of the PMT gain 


\section{Chapter 1}

\section{Introduction}

About half of all Canadians will be diagnosed of cancer in their life time, and $25 \%$ of Canadians

will die of cancer [1]. Cancer is now the leading cause of Canadian death, accounting for $30 \%$ of all death in Canada. Half of the cancer patients will receive radiation therapy. Most of those who receive radiotherapy will be treated with external radiation beam. In external beam radiotherapy, a linear accelerator (Linac) is used to produce and aim high-energy (megavoltage or MV) x rays from outside the body into the tumor. The treatments are usually delivered in daily fractions over several weeks. High-energy $\mathrm{x}$ rays will kill not only tumor cells, but also healthy cells. The goal of radiotherapy is to eradicate the tumor while sparing surrounding healthy tissues as much as possible. To achieve this goal, the tumor position must be accurately localized before the radiation treatment is delivered [2].

In this chapter, the importance of MV x-ray imaging for image-guided radiotherapy, the principle of MV x-ray imaging, current challenges with MV x-ray detection and research progress in developing MV x-ray detectors are briefly introduced.

\section{$1.1 \quad$ Importance of MV x-ray imaging}

With the advance of modern imaging technologies, such as computed tomography (CT), magnetic resonance imaging (MRI) and positron emission tomography (PET) the accurate delineation of complex tumor volume in 3D space can be achieved. It allows us to shape and deliver radiation beams that are highly conformal to the tumor with a high dose gradient at the boundary to achieve the treatment goal [3]. However, a number of complicating factors make the delivery of a treatment as planned a non-trivial task. First of all, the tumor position relative to the patient's anatomy may change from the time when the tumor is imaged to the time when the treatment is delivered. In addition, the size and shape of a tumor may vary during 
a treatment course. Moreover, treatment setup may introduce localization errors. Finally, the tumor position may even change within one dose fraction. Due to the high dose gradient near the boundary of the treated volume, missing the target means failure in treatment with severe side effects to healthy tissues. It is therefore crucial to accurately locate and verify the tumor position in the treatment room before a treatment plan can be successfully executed.

Image-guided radiotherapy (IGRT) can be achieved using portal imaging system, kilovoltage $(\mathrm{kV})$ cone beam computed tomography (CBCT) and MRI on a Linac Currently, kV CBCT is the most often used technique in the treatment room for tumor localization. Figure 1.1 shows a Linac machine with a $\mathrm{kV}$ CBCT system attached perpendicular to the treatment beam. The combination of MRI and Linac is new and the capital cost limits its widespread application. Portal imaging is the acquisition of images with a radiotherapy beam and an MV electronic portal imaging device (EPID),

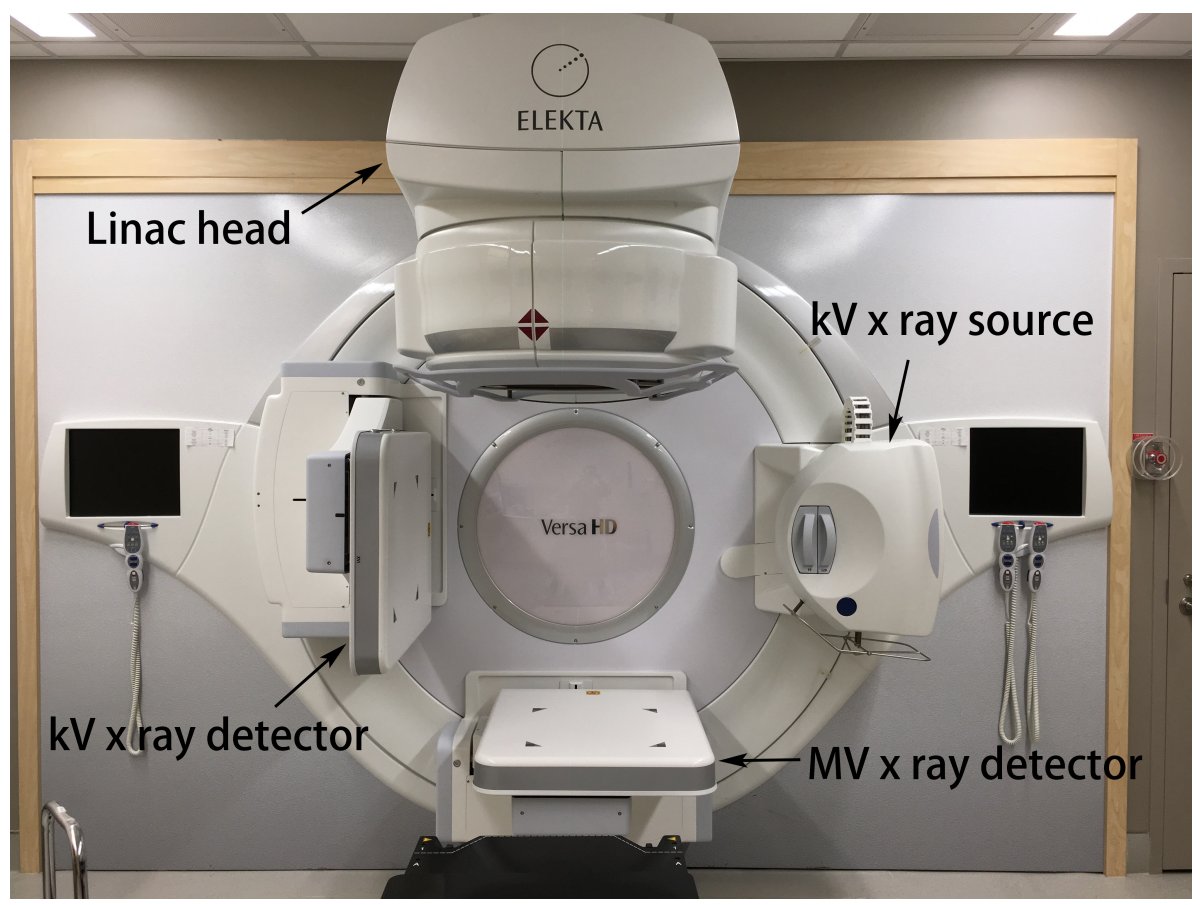

Figure 1.1: A Linac with a $\mathrm{kV}$ imaging system attached perpendicular to the treatment beam in the treatment room.

Although both $\mathrm{kV}$ CBCT and MRI provide better soft tissue contrast than portal imaging, MV portal imaging offers a unique benefit from the beam's eye view which shows exactly a projection of the volume treated. As a result, portal imaging system can be used to calibrate the spatial relation between the coordinate system of the Linac and that of the $\mathrm{kV}$ CBCT or 
MRI system [4,5]. In addition, MV imaging presents some distinct advantages. Compared to $\mathrm{kV}$ CT, MV imaging shows reduced artifacts due to the existence of high $\mathrm{Z}$ materials such as dental fillings and hip prostheses [6, 7]. The CT number acquired from MV CT can be readily used for dose calculations and inhomogeneity corrections in treatment planning, without the need for a conversion table as used for $\mathrm{kV}$ CT [8, 9. Moreover, MV portal imaging could be used for real time fluoroscopic imaging to monitor the tumor motion during the treatment [4]. It is therefore desirable to have a high quality MV x-ray imaging system in the treatment room for IGRT applications.

\section{$1.2 \quad$ Principle of MV x-ray imaging}

In x-ray imaging, when a uniformly distributed photon beam incident on an object, the object will attenuate a certain number of photons depending on the atomic number and density variation in the object (Fig. 1.2), resulting in a variation of photon distribution at the exit surface of the object. This represents the input image to the imaging system. The detector will then sense the distribution of $\mathrm{x}$ rays in the input image and display it as an output image. The output image quality is dependent on the number $\mathrm{x}$ rays that are captured by the detector to generate signals. Almost all of today's radiotherapy systems use flat-panel detectors for imaging. There are two basic types of amorphous silicon flat-panel detectors, i.e., direct and indirect converter types, depending on how incident $\mathrm{x}$ rays are converted into charges stored in the capacitive element as signals in each pixel [10].

The direct converter type detector uses a thick layer of photoconductor which is electrically coupled to a separate capacitor built into each pixel (Fig. 1.3 [10. In this approach, the energy of the incident $\mathrm{x}$ ray is converted into electron-hole pairs in the photoconductor layer and this imaging signal is stored in pixel capacitors until readout.

The indirect converter type detector consists of a metal plate (known as buildup) coupled to a scintillator which is positioned on photodiode arrays (Fig. 1.4 [10. Incident x rays are converted into detected signals in multiple steps. First MV x rays interact with the metal plate and produce energetic secondary electrons. These electrons then deposit their energies by either ionization or excitation in the scintillator. One secondary electron may excite hundreds or even thousands of atoms and produce a corresponding number of visible photons. Visible photons that travel in the direction of the photodiode will be absorbed and converted into electron-hole pairs. These charges are stored in the intrinsic capacitance of the photodiode until readout. The two dimensional distribution of charge signals at each pixel element form an image. Most of current EPID $\$$ in the clinic use indirect converter type detectors.

Energetic secondary electrons ejected from the interaction between incident photons and 


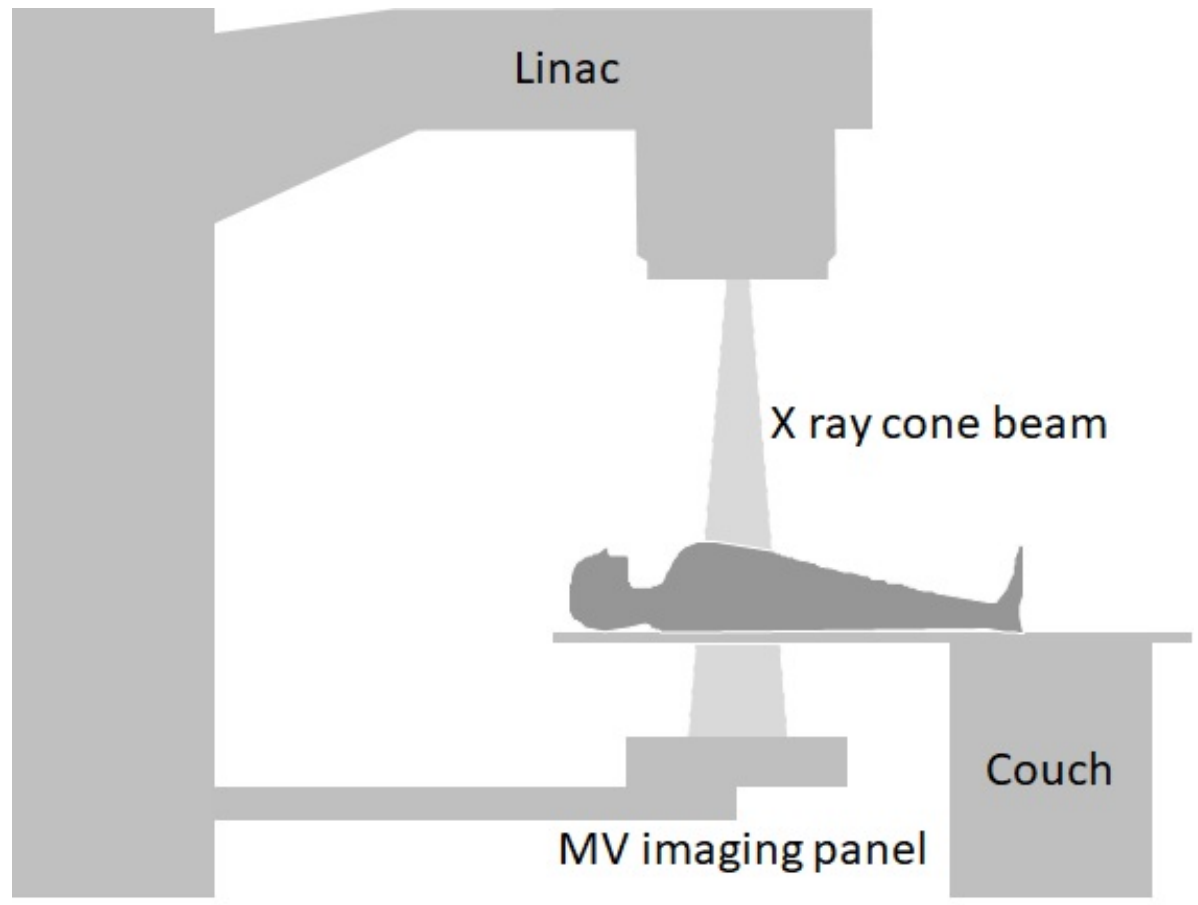

Figure 1.2: Illustration of the acquisition of a portal image.

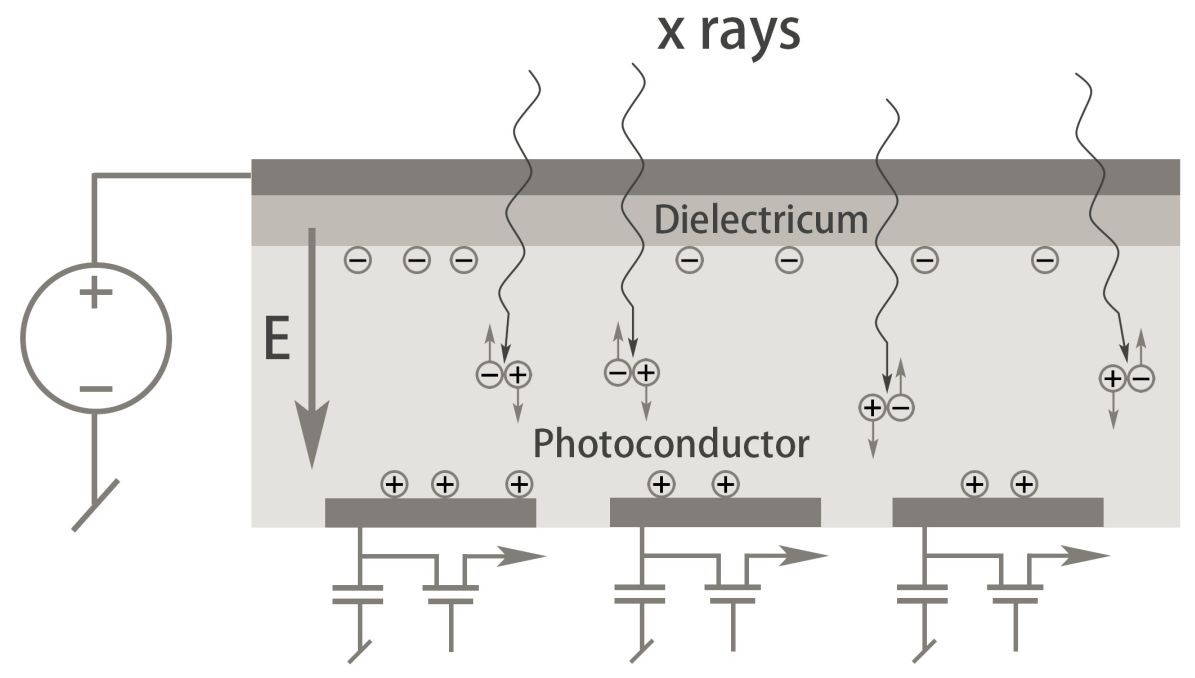

Figure 1.3: Illustration of working principle of a direct converter flat-panel detector. Adapted from Ref. 10 


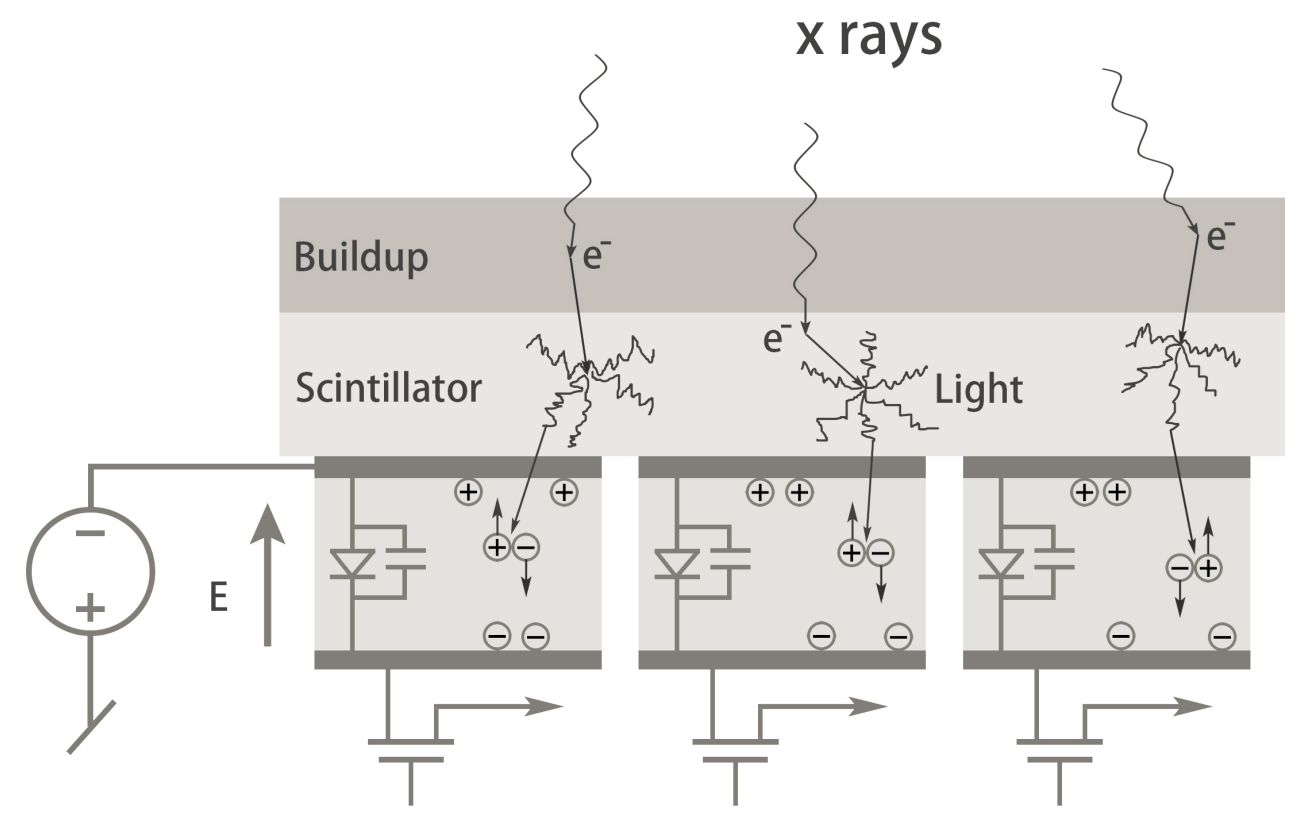

Figure 1.4: Illustration of working principle of an indirect converter flat-panel detector. Adapted from Ref. 10 
the detector will travel certain distances before depositing their energy. High-energy charged particles (electrons) lose their energy through multiple collisional processes. They can travel a substantial distance before depositing all of their energy through ionization, excitation and radiation losses. Therefore, the maximum energy deposition is not at the surface of the detector, but at the distance approximately equal to the range of the secondary electrons. To maximize the number of detected incident photons, the ideal point of signal collection is where the energy deposition peak is located. This is why a metal plate buildup is placed on top of the scintillator. The metal plate also blocks scattered $\mathrm{x}$ rays incident on the detector which would otherwise lower the image contrast 11 .

\subsection{Important physical processes in x-ray detection}

To generate detectable signals, incident $\mathrm{x}$ rays have to interact with detecting materials. Since the interaction probability of $\mathrm{x}$ rays with matter depends on the $\mathrm{x}$-ray energy, it is necessary to clarify how x-ray energy is specified in clinical diagnostic imaging and radiotherapy. The most commonly used mechanism to produce $\mathrm{x}$ rays for clinical applications is bremsstrahlung (breaking) radiation [12]. When an electron with high kinetic energy passes in the vicinity of a nucleus, it may suffers a sudden deflection and deceleration due to the Coulomb force from the nucleus. As a result, a part or all of its energy will be lost as electromagnetic radiation (x rays). As many such events occur for each incident electron with each event resulting in a different energy loss, the x-ray emission covers a broad range of energies.

In diagnostic imaging systems, x-ray tubes are used as the x-ray source. An x-ray tube is made of an evacuated glass envelope which encapsulates a cathode (filament) and an anode 13 . When passing through a current (typically between 3-6 A), the filament is heated up to a very high temperature and some of the electrons in the filament will gain enough thermal energy to be emitted out. The electric field generated by the high voltage (typically in the range 20-150 $\mathrm{kV}$ ) between the anode and cathode will accelerate the electrons to collide into the anode and produce bremsstrahlung radiation. The x-ray energy produced by an x-ray tube is indicated by the electron accelerating voltage (e.g., $\mathrm{kVp}$ ).

In a modern Linac for radiotherapy, electrons are accelerated using high-frequency electromagnetic waves through a specially designed linear tube called waveguide 13 . The photon beam energy is specified in term of nominal accelerator potential (4-25 MV). Figure 1.5 shows the x-ray spectrum of $6 \mathrm{MV}$ treatment planning beam (Pinnacle3, Philips, Fitchburg). The average photon energy of the $6 \mathrm{MV}$ beam is about $2 \mathrm{MeV}$.

In the energy range of the treatment beam, the most important three processes of $\mathrm{x}$ rays interacting with matter are photoelectric effect, Compton scattering and pair production 12 . 


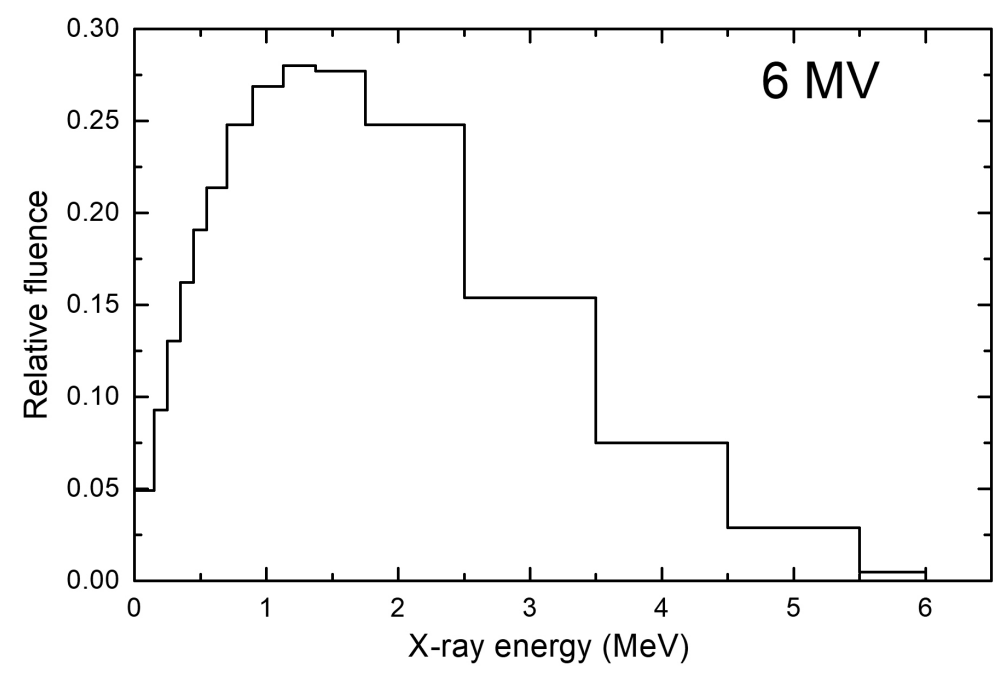

Figure 1.5: X-ray spectrum generated by a treatment planning system at $6 \mathrm{MV}$ (Pinnacle3, Philips, Fitchburg).

In the photoelectric effect, a photon is absorbed by an atom and as a result one of the orbital electron is ejected. The kinetic energy of the ejected electron (photoelectron) is equal to the energy of the incident photon minus the binding energy of the orbital electron. In the Compton process, the photon interacts with an atomic electron as though it were a "free" electron, that is, the photon energy is much greater than the binding energy of the electron. The electron then receives some of the energy from the photon and is ejected at an angle $\theta$ relative the incident photon direction. The photon is scattered at an angle $\phi$ with reduced energy. When the incident photon energy exceeds $1.02 \mathrm{MeV}$, two times the rest energy of an electron, pair production may occur. In this process, the photon interacts with the electromagnetic field of an atomic nucleus and gives up all its energy creating an electron-positron pair.

One parameter that describes the probability of incident photons interacting with matter is linear attenuation coefficient, $\mu$. Suppose that $N_{0}$ photons are incident on a detector and the number of transmitted photons is $N$ as illustrated in Fig. 1.6. we have 13]

$$
N=N_{0} \mathrm{e}^{-\mu d}
$$

where $d$ is the detector thickness. $N$ is the number of photons that are not detected which represent lost information. As $\mu d$ gets larger, $N$ gets smaller.

The linear attenuation coefficient has a unit of length ${ }^{-1}$ (e.g., $\left.\mathrm{cm}^{-1}\right)$. It depends on the density of the material since the attenuation produced by a thickness $d$ depends on the number 


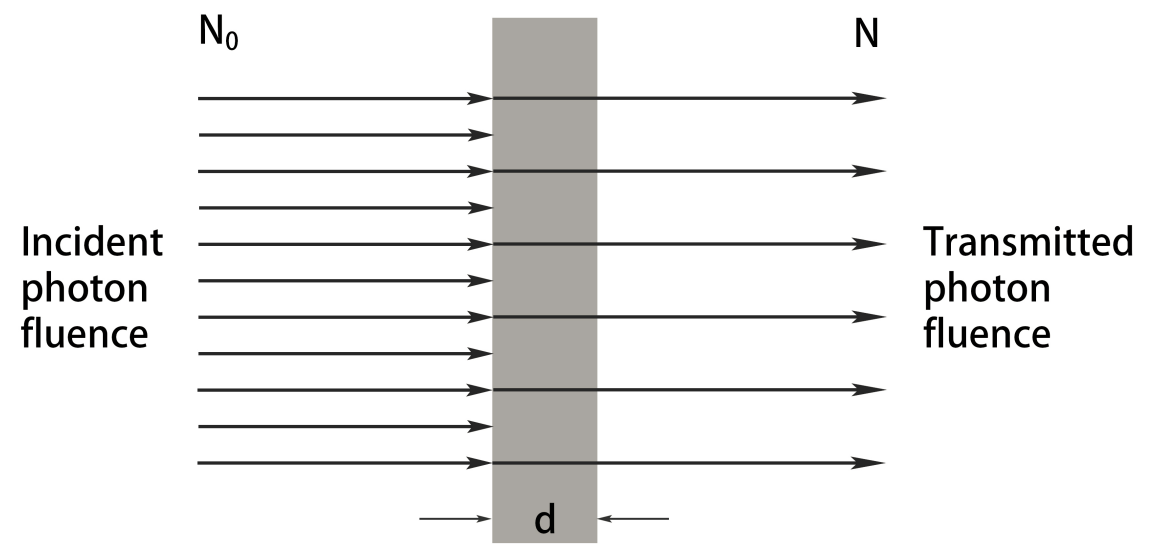

Figure 1.6: Illustration of photon fluence attenuated by detecting material.

of electrons in that thickness. By factoring out density $\rho$ from $\mu$, the resulting coefficient $\mu / \rho$, known as mass attenuation coefficient, will be independent of density. Thus, mass attenuation coefficient has a unit of $\mathrm{cm}^{2} / \mathrm{g}$ and depends on the nature (atomic composition) of the material.

The probabilities of photoelectric, Compton, and pair production processes depend on the incident photon energy and the detector material. Figure 1.7 shows the mass attenuation coefficients for these processes and the total mass attenuation coefficient as a function of incident photon energy in copper [14], a common buildup material. In the therapeutic energy range, Compton scattering is the dominant interaction process 13 . The probability of Compton interaction depends on the election density, i.e., the number of electrons per unit volume, of the material. It is independent of the atomic number $\mathrm{Z}$ per unit mass. It is proportional to the density and atomic number $\mathrm{Z}$ per unit volume with the exception of hydrogenated material. The electron density in hydrogenous material is almost doubled to that of anhydrogenous materials 13 . 


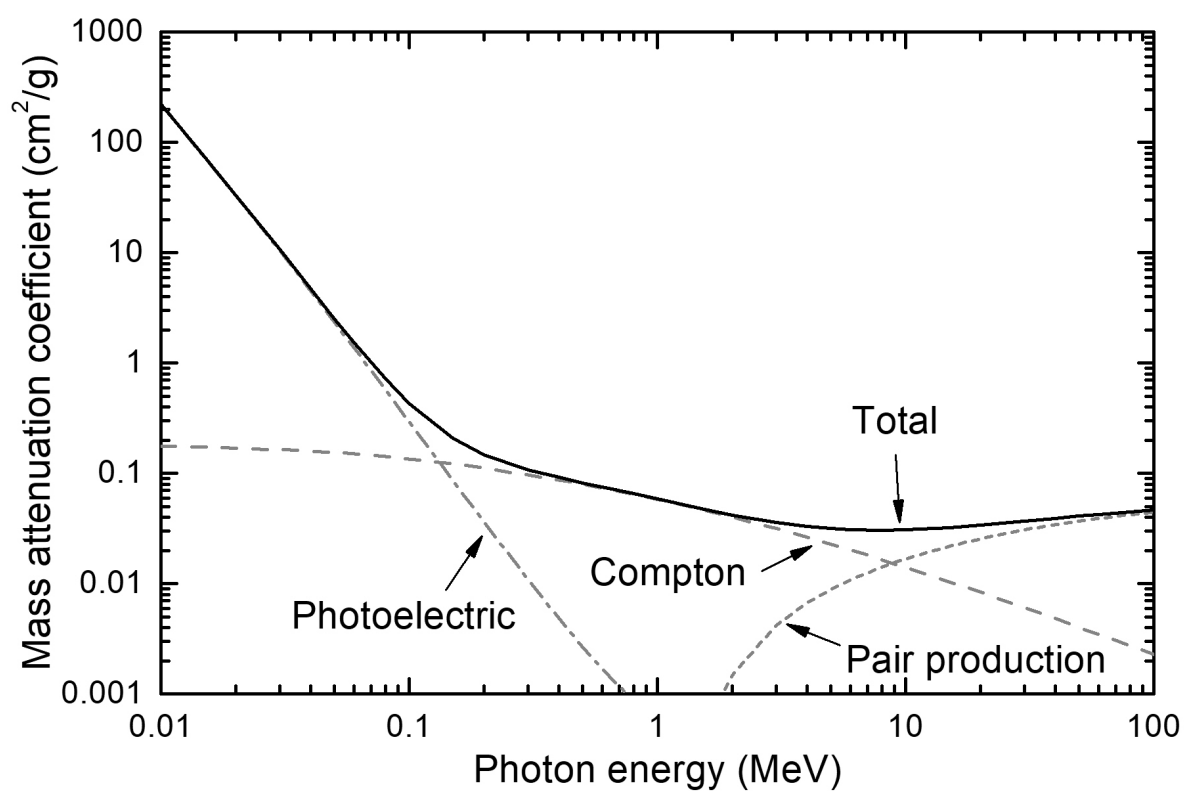

Figure 1.7: Mass attenuation coefficients due to photoelectric effect, Compton effect and pair production for $\mathrm{x}$ rays in copper. Solid line is the sum of three mass attenuation coefficients. Data source: Ref. [14].

\subsection{Parameters that characterize the quality of x-ray images and the performance of imaging systems}

\subsubsection{Contrast}

Contrast, C, describes how an object can be differentiated from its background and is defined as

$$
\mathrm{C}=\frac{\text { signal }}{\text { mean signal }}=\frac{\phi_{p_{2}}-\phi_{p_{1}}}{\left(\phi_{p_{2}}+\phi_{p_{1}}+\phi_{s}\right) / 2}
$$

where $\phi_{p_{1}}, \phi_{p_{2}}$ are the primary photon fluences passing through the normal tissue and the lesion region and reaching the image detector, respectively. $\phi_{s}$ is the scattered photon fluence (Fig. 1.8. If we image a bone in water phantom, it has been shown that the contrast can also be expressed as 15

$$
\mathrm{C}=\frac{2\left(1-\mathrm{e}^{-\triangle}\right)}{1+\mathrm{e}^{-\triangle}+\frac{2 \mathrm{SF}}{1-\mathrm{SF}}},
$$


where $\triangle=L_{x}\left|\mu_{\text {bone }}-\mu_{\text {water }}\right|, L_{x}$ is the thickness of the anatomic structure (Fig. 1.8), $\mu_{\text {bone }}$ and $\mu_{\text {water }}$ are the x-ray attenuation coefficients for bone and water, respectively and $S F=$ $\phi_{s} /\left(\phi_{s}+\phi_{p}\right)$ is the scatter fraction. In detecting tumor, the contrast is determined by the difference in x-ray attenuation between the normal tissue and the lesion. As mentioned above, Compton scattering depends on the electron density of a material. The normal tissue and the lesion may not have highly different electron densities. Also, Compton scattering cross section decreases with incident photon energy (Fig. 1.7). The contrast drops significantly in MV x-ray imaging.
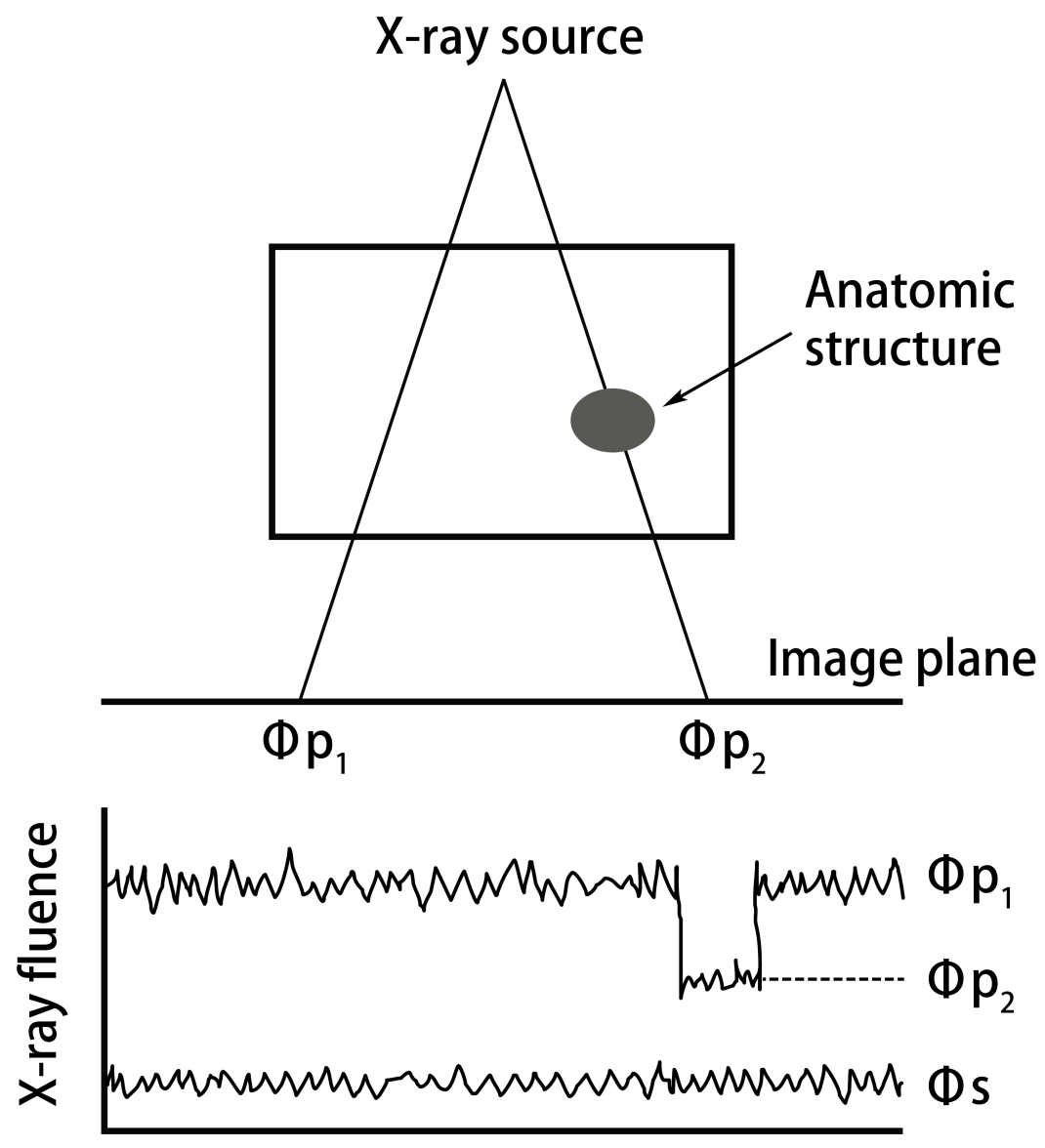

Figure 1.8: Schematic representation of the portal imaging process. $\phi$ is x-ray fluence. Adapted from Ref. 15. 


\subsubsection{Signal to noise ratio (SNR)}

A limiting source of noise in x-ray imaging is x-ray quantum statistics. The imaging formation involves interaction between the detector and the x-ray quanta, which is associated with a statistical uncertainty known as x-ray quanta mottle. The number of x rays detected in certain time interval follows Poisson distribution, in which the variance of the number of $x$ rays detected is equal to the average number of detected $\mathrm{x}$ rays. Therefore, the SNR can be calculated as 15

$$
\mathrm{SNR}=\frac{\text { image signal }}{\text { noise }}=\frac{\phi_{p_{2}}-\phi_{p_{1}}}{\sqrt{\left(\phi_{p_{2}}+\phi_{p_{1}}+2 \phi_{s}\right) / 2}} .
$$

Similar to the expression of contrast in Eq. 1.3. we have 15

$$
\mathrm{SNR}=\sqrt{A \phi_{i} T_{\mathrm{x}} \eta_{d}} \frac{2\left(1-\mathrm{e}^{-\triangle}\right)}{\sqrt{1+\mathrm{e}^{-\triangle}+\frac{2 \mathrm{SF}}{1-\mathrm{SF}}}},
$$

in which $A$ is the area of the detector, $\phi_{i}$ is the incident fluence, $T_{\mathrm{x}}$ is the fraction of transmitted $\mathrm{x}$ rays, and $\eta_{d}$ is the detector efficiency. $A \phi_{i} T_{\mathrm{x}} \eta_{d}$ is the total number of x rays detected. As can be seen from Eq. 1.5, the SNR is proportional to the number of $\mathrm{x}$ rays detected.

Another noise source is scattered $x$ rays. As scattering of $x$ rays in the patient's body is random, scattered $\mathrm{x}$ rays do not carry the anatomic information and add to the noise. To improve the image quality, we would like to improve the SNR as much as possible. Unfortunately, due to the low probability of interaction of high energy $\mathrm{x}$ ray with matter, the fraction of $\mathrm{x}$ rays can be detected is very low. To get a reasonable SNR, currently an elevated dose has to be used in portal imaging compared to clinical diagnostic imaging.

\subsubsection{Spatial resolution}

In the spatial domain, the spatial resolution of a detector can be characterized by its point spread function (PSF) 16. The PSF is the image of a point object formed by the detector. It describes how the object is blurred by the imaging system. To measure a PSF a tiny hole in a thick layer of lead can be used to expose the detector to a narrow beam of $\mathrm{x}$ rays. However, the measurement of PSF can be difficult for some imaging systems. Instead, line spread function (LSF) can be measured. The LSF can be considered as the superposition of PSFs of a larger number of point sources along a line. To determine the LSF, a slit object is imaged to generate a profile perpendicular to the slit object. Mathematically, we have 16

$$
\operatorname{LSF}(x)=\int_{-\infty}^{\infty} \operatorname{PSF}(x, y) \mathrm{d} y .
$$


In the frequency domain, the modulation transfer function (MTF) is a complete description of the resolution of an imaging system. The MTF illustrates how an object's contrast is reduced by the imaging system as a function of the object size (i.e., spatial frequency). Mathematically [17,

$$
\operatorname{MTF}(x, y)=\frac{|\mathrm{T}(u, v)|}{\mathrm{T}(0,0)}
$$

where $\mathrm{T}(u, v)$ is the characteristic function of the imaging system. i.e., the Fourier transform of the PSF,

$$
\mathrm{T}(u, v)=\iint_{-\infty}^{\infty} \operatorname{PSF}(x, y) \mathrm{e}^{-i 2 \pi(u x+v y)} \mathrm{d} x \mathrm{~d} y .
$$

It can be shown, the MTF in one direction can be expressed as

$$
\operatorname{MTF}(u)=\int_{-\infty}^{\infty} \operatorname{LSF}(x) \mathrm{e}^{-i 2 \pi u x} \mathrm{~d} x
$$

Normally the spatial frequency in the unit of line pairs $/ \mathrm{cm}\left(\mathrm{lp} / \mathrm{cm}\right.$ or simply $\left.\mathrm{cm}^{-1}\right)$ at $50 \%$ MTF(0) is reported as a measure of the resolution of an imaging system.

\subsubsection{Quantum efficiency (QE) and detective quantum efficiency (DQE)}

In this work, we define $\mathrm{QE}$ as the percentage of $\mathrm{x}$ rays that are attenuated by the detector, that is, $\mathrm{QE}=\frac{N_{0}-N}{N_{0}}$, as illustrated in Fig. 1.6. It should be noted that $\mathrm{x}$ rays attenuated may not be detected.

The DQE is a measure of the fraction of incident quanta that actually contribute the image SNR. It describes the overall SNR performance of the system and can be defined as 11

$$
\mathrm{DQE}=\frac{\mathrm{SNR}_{\text {out }}^{2}}{\mathrm{SNR}_{\text {in }}^{2}} .
$$

A practical expression of measuring DQE experimentally is given by 16

$$
\operatorname{DQE}(u)=\frac{\bar{d}^{2} \operatorname{MTF}^{2}(u)}{\bar{q} \operatorname{NPS}(u)},
$$

where $\bar{d}$ is the mean detector signal; $\bar{q}$ is the mean incident x-ray fluence; and $\operatorname{NPS}(u)$ is the noise power spectrum (NPS) that characterizes the noise properties of a system. 
For a $2 \mathrm{D}$ detector, $\operatorname{NPS}(u)$ is the NPS evaluated in one direction and is given by 16

$$
\operatorname{NPS}(u)=\lim _{X \rightarrow \infty} \frac{1}{X}\left|\int_{X} \triangle d(x) \mathrm{e}^{-i 2 \pi u x} \mathrm{~d} x\right|^{2}
$$

where $\triangle d(x)=d(x)-\overline{d(x)}$ is the noise at $x . d(x)$ is the detector signal at $x$ and $\overline{d(x)}$ is the mean value of $d(x)$.

\subsection{Current challenges with MV x-ray imaging}

For x-ray detection, photoelectric effect dominates at low incident x-ray energies, while Compton scattering dominates at high x-ray energies (Fig. 1.7). The clinical diagnostic imaging utilizes a $\mathrm{kV}$ x-ray source and detector which has a much higher DQE than the MV x-ray imaging system. It is desirable to have a $\mathrm{DQE}$ as close to 1 as possible. To increase the portion of incident $\mathrm{x}$ rays detected, we have to increase the thickness of a given detecting material, since the probability of the Compton process is proportional to the number of electrons presented in the detector. Due to the fact that the range of secondary electrons produced by the $6 \mathrm{MV}$ beam in copper is about $1.5 \mathrm{~mm}$, further increasing the buildup thickness will not result in more secondary electrons traveling into the scintillator. If the thickness of the scintillator is increased, the resolution degrades quickly because of the divergence of the emitted lights. To get a reasonable resolution, the copper plate/scintillator thickness is limited to approximately $2 \mathrm{~mm}$. The QE of MV EPID is typically only 2-4\%, as compared to the theoretical limit of $100 \%$, and the DQE is only approximately 1\% 18. As a result, the dose required to obtain a clinically useful image is unreasonably high. Studies have shown that a DQE(0) of $20 \%$ is desirable to achieve acceptable image quality at acceptably low doses [2, 19,20 .

\subsection{Research progress in developing MV x-ray detectors}

To improve the quality of portal imaging, high efficiency MV x-ray detectors have been widely investigated $[2,21,32]$. The current research in this field focuses on developing 2D pixelated scintillator matrices. One approach involves the use of thick, segmented 2D scintillator crystals in which crystalline scintillators are sliced in two perpendicular directions to form $2 \mathrm{D}$ array of small pixels $20,27,28,31,32$. These scintillating pixels are separated by optically opaque and reflective septal walls. The opaque/reflective septal walls prevent light produced within a pixel from propagating into neighboring pixels and degrading the spatial resolution. Therefore, thicker detecting materials can be used to increase the QE.

A variety of scintillating materials, such as cesium iodide (CsI) 20, 25, bismuth germanate 
(BGO) 31, 33, sintered gadolinium oxysulfide (GOS) 34 and cadmium tungstate (CWO) [35, 36, have been explored to make pixelated x-ray detectors. GOS is commonly used in diagnostic CT detector 37. It is also currently used in MV x-ray detectors as the phosphor screen. Its low optical transmissivity limits the gain that can be obtained by increasing its thickness. A pixelated ceramic GOS array with an area of $40 \times 10 \mathrm{~cm}^{2}$ and a thickness of $1.8 \mathrm{~mm}$ was studied for imaging at $6 \mathrm{MV} 34$. Its sensitivity was only 2.5 times that of the $\mathrm{Cu} /$ phosphor screen detector.

Seppi et al. built the first large area high QE MV x-ray detector using pixelated thalliumdoped CsI scintillator coupled to a flat-panel imager 25. The detector had an area of $40 \times 30 \mathrm{~cm}^{2}$ and a thickness of $9 \mathrm{~mm}$. Comparing to the conventional phosphor screen, the sensitivity was significantly increased. However, the DQE(0) at $6 \mathrm{MV}$ was still less than $10 \%$ and due to optical cross talk between neighboring pixels, the spatial resolution was only about half of that provided by the phosphor screen. They concluded that the imager was not suitable for general image guidance in radiotherapy. Sawant et al. later built a $40 \mathrm{~mm}$ thick pixelated CsI imager and improved the $\mathrm{DQE}(0)$ at $6 \mathrm{MV}$ to $22 \%[20]$. However, the spatial resolution of their imager was even inferior to that of Seppi's because as the detector thickness increases, the spread out of lights in the lateral direction also increases due to the sharing of light between pixels, and the scintillating pixels were not well aligned.

Bismuth germanate was shown to be a preferred scintillator due to its higher density and higher index of refraction 31,33. To achieve the same QE the detector can be made thinner when the density of the material is higher to reduce the degradation of the spatial resolution. Higher index of refraction increases the probability of total internal reflection that optical photons undergo at the pixel-glue interface and therefore reduces the cross talk. However, these advantages of BGO are undermined by its low light output and requirement of preirradiation with 2000 cGy to achieve acceptable output stability.

Cadmium tungstate has many desired properties as a MV x-ray detecting material, such as a high density, $7.9 \mathrm{~g} / \mathrm{cm}^{3}$, and a high index of refraction, $\mathrm{n}=2.3$. Comparing to BGO, CWO has twice the light output; and comparing to GOS ceramic, CWO is highly transparent 32, 35, 36. A 1D piecewise-focused CWO array with a thickness of $10 \mathrm{~mm}$ was built and tested, and a DQE(0) of $19 \%$ was achieved at $6 \mathrm{MV}$ [35]. The reconstructed CT image had a resolution of $5 \mathrm{lp} / \mathrm{cm}$ at the isocenter (ISO), Recently, Star-Lack et al. built a 2D pixelated CWO array which is piecewise focused with a thickness of $15 \mathrm{~mm} 32$. The measured DQE(0) was $22 \%$ and spatial resolution $7 \mathrm{lp} / \mathrm{cm}$. However, the $\langle 010\rangle$ cleave plane inherent to the crystal structure of the CWO makes it difficult to machine and polish for making pixels with a high aspect ratio. The cost of making such detectors is very high.

Another approach involves the use of a stack of four conventional MV x-ray detection 
layers 38 41. Each layer consists of a copper plate, a scintillator (GOS) layer and a low noise image readout system. By combining signals from all four layers, a DQE(0) of about $6.7 \%$ was observed and the SNR was increased by a factor of $1.7[38$. A limitation of this approach might be the degradation in $\mathrm{MTF}$ if more layers are used to further increase the $\mathrm{DQE}$, due to the defocusing caused by beam divergence.

Pang and his group have been investigating new approaches that could improve the QE of EPID $\beta$ by more than an order of magnitude while maintaining spatial resolution comparable to current EPIDs 26, 42,47. One approach involved the use of micro gas chamber. Each gas chamber had a width of less than $1 \mathrm{~mm}$ and a length (the thickness of the detector) of $22 \mathrm{~mm}$ 26, 43. The chamber wall were made of high density metals such as copper and tungsten to increase the $\mathrm{QE}$ and improve spatial resolution. The properties of a single pixel prototype detector were measured and a $\mathrm{QE}$ of $66 \%$ at $6 \mathrm{MV}$ was achieved. The measured spatial resolution was $0.3 \mathrm{lp} / \mathrm{mm}$ which is about the same as current EPIDs [43].

Another approach was based on Cerenkov radiation, subsequently referred to as Cerenkov portal imaging detector (CPID) $[44,46,48,49]$. Cerenkov radiation is the light emitted when a charged particle travels through a dielectric medium at a speed greater than the phase velocity of light in that medium. The CPID consisted of a large area and thick (non-scintillating) fiberoptical taper directly coupled to an optically sensitive flat-panel imager. A small prototype CPID was built and low dose MV images were obtained. It was discovered that the CPID is an inherent anti-scatter detector, the first of this kind, for MV x-ray imaging, due to the fact that scattered $\mathrm{x}$ rays have lower energy and are less likely to excite secondary electrons with high enough energy to produce Cerenkov radiation 46,50. However, it was found that a large area (e.g., $40 \times 40 \mathrm{~cm}^{2}$ ) CPID would require a technology (i.e. an imager with an avalanche gain) that is not yet available because of the low light yield of Cerenkov radiation.

\subsection{Objective of this project}

The objective of this project is to develop an MV x-ray detector with a QE one order of magnitude higher than that of the current EPIDs in the clinic and a comparable spatial resolution. In this work, we use plastic scintillating fibers to replace the non-scintillating fibers to overcome the weaknesses of the CPID and use lead as the septal material. Plastic scintillating fibers will significantly improve the radiation induced light output and thus a conventional flat-panel imager (without an avalanche gain) will be sufficient to build a high QE EPID. As the light produced in the fiber core and within the acceptance angle of the fiber will be guided to the detecting device by total internal reflection (Fig. 1.9), the detector can be made thicker to increase the $\mathrm{QE}$ and at the same time maintain acceptable spatial resolution. 


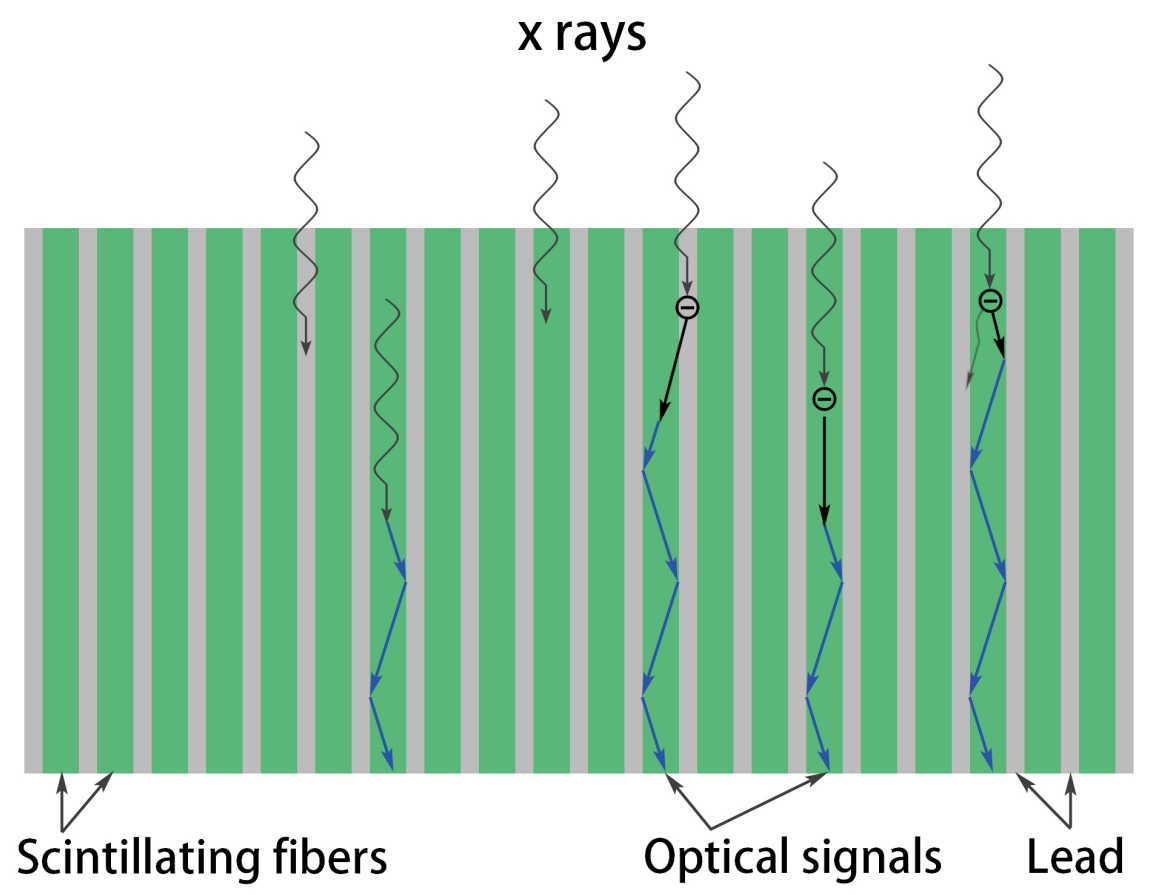

Figure 1.9: Illustration of the signal production and conduction in the proposed detector. 


\section{Chapter 2}

\section{Materials and methods}

The prototype detector was made by embedding scintillating fibers (Kuraray SCSF-78MJ, Kuraray Co., Ltd., Tokyo, Japan) in lead to form a honeycomb pattern. Very thin corrugated lead sheets and scintillating fibers were bonded together using BC-600 optical epoxy (Saint-Gobain Crystals, OH, USA). A diagram of the detector (top view) is shown in Fig. 2.1 and a picture of the prototype detector placed on the "Ryerson University" logo is shown in Fig. 2.2 .

Kuraray SCSF-78MJ is a double-clad fiber with a diameter of $1 \mathrm{~mm}$. Materials that are used to make the fiber and their properties are listed in Table 2.1. When the detector was coupled to a flat-panel imager, the signal was low, probably due to the lead contamination on the fiber ends introduced during the detector fabrication process.

To measure the property of the detector, a fiber near the center of the detector was removed and a longer fiber was inserted into the detector. The other end of the longer fiber was coupled to a $10 \mathrm{~m}$ long optical fiber through a fiber optic dual switch (FOS-2×2-TTL, Ocean Optics, Inc. Dunedin, FL, USA). The optical fiber was connected to a photomultiplier tube (PMT) (R6095, Hamamatsu Photonics K.K., Japan) which was placed outside the treatment room to minimize the signal due to direct interaction between the radiation and the PMT. Detector property measurements were carried out at the Odette Cancer Centre, Sunnybrook Health

Table 2.1: Material properties of the Kurary SCSF-78MJ scintillating fiber.

\begin{tabular}{llcc}
\hline \hline & Material & Refractive index, $\mathrm{n}$ & Density $\left(\mathrm{g} / \mathrm{cm}^{3}\right)$ \\
\hline Core & Polystyrene (PS) & 1.59 & 1.05 \\
\hline Inner cladding & Polymethylmethacrylate (PMMA) & 1.49 & 1.19 \\
\hline Outer cladding & Fluorinated polymer (FP) & 1.42 & 1.43 \\
\hline \hline
\end{tabular}




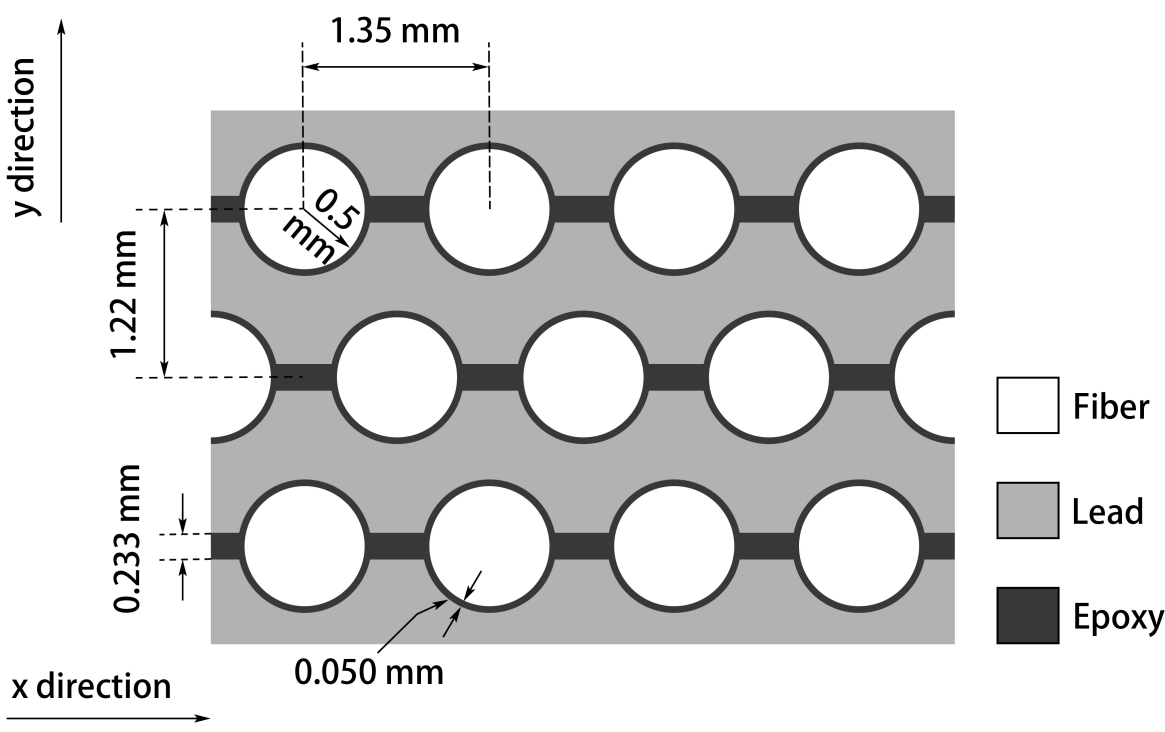

Figure 2.1: Diagram of the prototype detector structure.

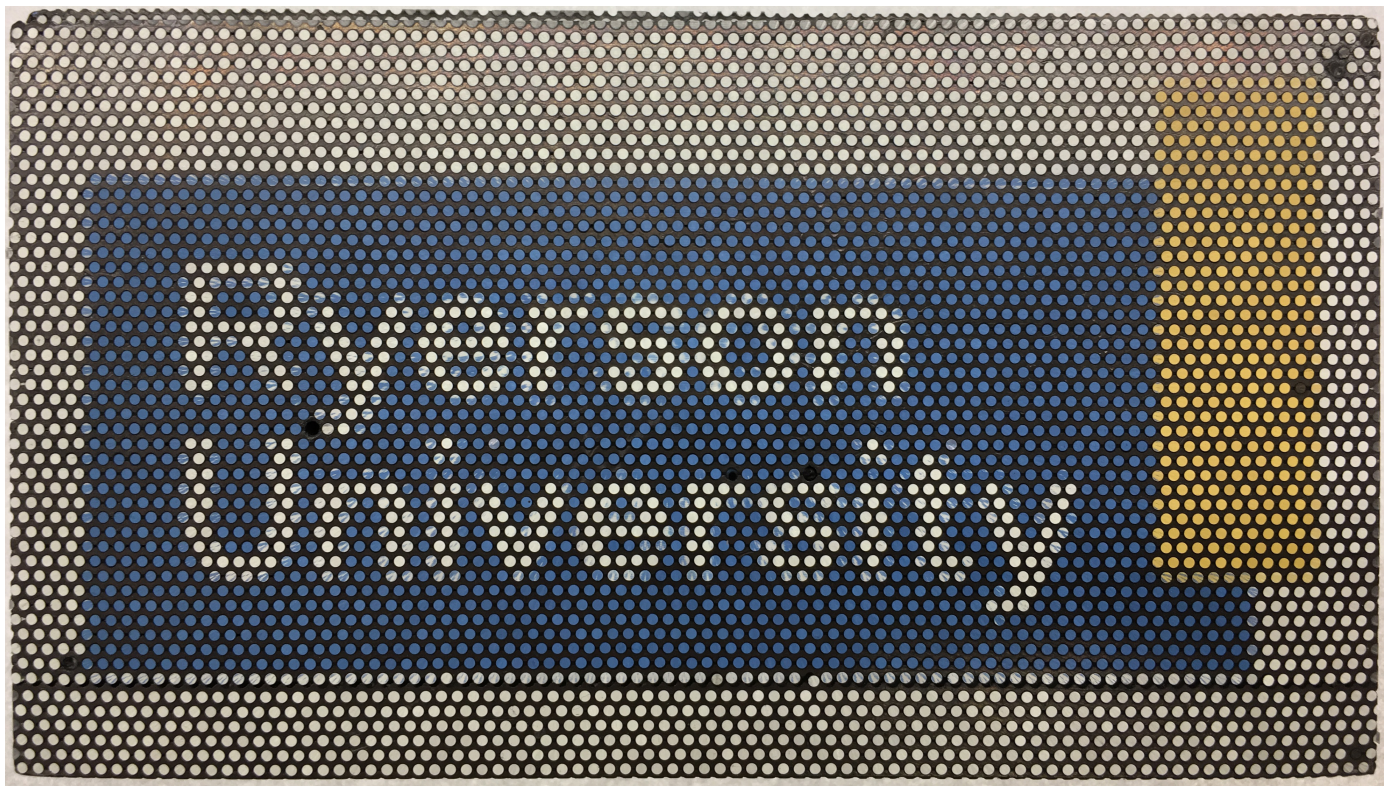

Figure 2.2: The prototype detector placed on top of the "Ryerson University" logo. 
Sciences Centre in Toronto, using a 6 MV beam generated from a Linac machine. The Linac was calibrated to deliver 1 cGy per monitor unit (MU) in water at a source to axis distance (SAD) of $100 \mathrm{~cm}$ and a depth of $d_{\max }=1.5 \mathrm{~cm}$ at a field size of $10 \times 10 \mathrm{~cm}^{2}$. SAD is the distance from the x-ray source in the Linac head to the gantry rotation axis. All field size referred to in this work are defined at the ISO, which is the point where the rotational axes of the Linac gantry, collimator and treatment couch meet. The collimator angle was set to 0 . The dose referred to in the work is the dose to water at a depth of $d_{\max }=1.5 \mathrm{~cm}$ with $\mathrm{SAD}$ of $100 \mathrm{~cm}$ setup.

\subsection{Scintillating fiber emission spectrum}

As visible lights generated in scintillating fibers upon irradiation will be sensed by photodiodes and converted to an output signal, the magnitude of the output signal will depend on the emission spectrum of the fibers. The fiber emission spectrum was measured using a FLAME-T spectrometer (Ocean Optics, Inc., Largo, FL, USA). One end of the fiber was connected to the spectrometer. The rest of the fiber was rolled into a circular shape with a diameter of about 15 $\mathrm{cm}$ and placed on the solid water which seated on the treatment couch. Then a $1.5 \mathrm{~cm}$ thick solid water buildup was placed on top of the fiber. The fiber was kept at the ISO during the measurement. A black cloth was used to cover the fiber and all lights in the room was turned off to prevent ambient light from entering the fiber and being measured by the spectrometer.

\subsection{Signal attenuation due to the optical dual switch and opti- cal fiber}

Since the signal generated in the scintillating fiber is sent to the PMT through an optical switch

and a 10 meter long optical fiber, there is a signal loss due to the reflection at the interface between the scintillating fiber and the optical switch and the attenuation in the optical fiber. To determine the percentage of the signal loss, the emission spectrum of a tungsten halogen light source (Model LS-1-CAL, Ocean Optics, Inc., Largo, FL, USA) was measured using the spectrometer as shown in Fig. 2.3. The signal in measurement 2 divided by that in measurement 1 is the ratio of the signal generated in the scintillating fiber and detected by the PMT.

\subsection{Linearity}

Ideally the response of the detector to a large range of radiation doses should be linear. To measure the linearity, another fiber that was $2 \mathrm{~cm}$ shorter than the fiber inserted into the 


\section{Measurement 1}

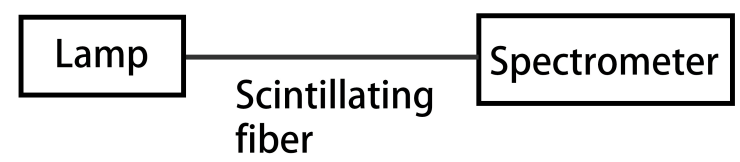

\section{Measurement 2}

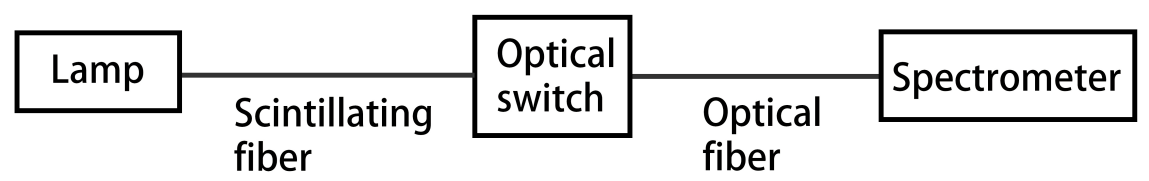

Figure 2.3: Diagram showing the measurement of signal attenuated by the optical switch and the optical fiber.

detector was running beside the detector fiber (Fig. 2.4). To get the signal due to only the segment of the fiber in the detector, the signal difference, i.e. the signal from detector fiber minus the signal from reference fiber, was measured. The radiation dose was varied in the range from 10 to 250 cGy to investigate the dependence of the output signal on the dose.

\subsection{Quantum efficiency}

To measure the QE the detector was placed on the couch at the ISO and an ionization chamber (Farmer model A19) at $50 \mathrm{~cm}$ away from the beam exit side of the detector to minimize the effect of scattered $\mathrm{x}$ rays generated in the detector. Ionization chamber readings were recorded at a fixed exposure with and without the presence of the detector in the x-ray beam. The field size at the ISO was varied from $2 \times 2 \mathrm{~cm}^{2}$ to $6 \times 6 \mathrm{~cm}^{2}$ to check if there is any dependence of QE on x-ray field sizes.

\subsection{Spatial resolution}

To characterize the spatial resolution of the detector, MTF was obtained from the Fourier transform of the LSF. To measure the LSF, a line field was generated by passing the x-ray beam through a slit made of two steel blocks, each with dimensions of $3.5 \mathrm{~cm} \times 7 \mathrm{~cm} \times 10.5 \mathrm{~cm}$. The surfaces of $7 \mathrm{~cm} \times 10.5 \mathrm{~cm}$ of two steel blocks were squeezed together with shims of 80 


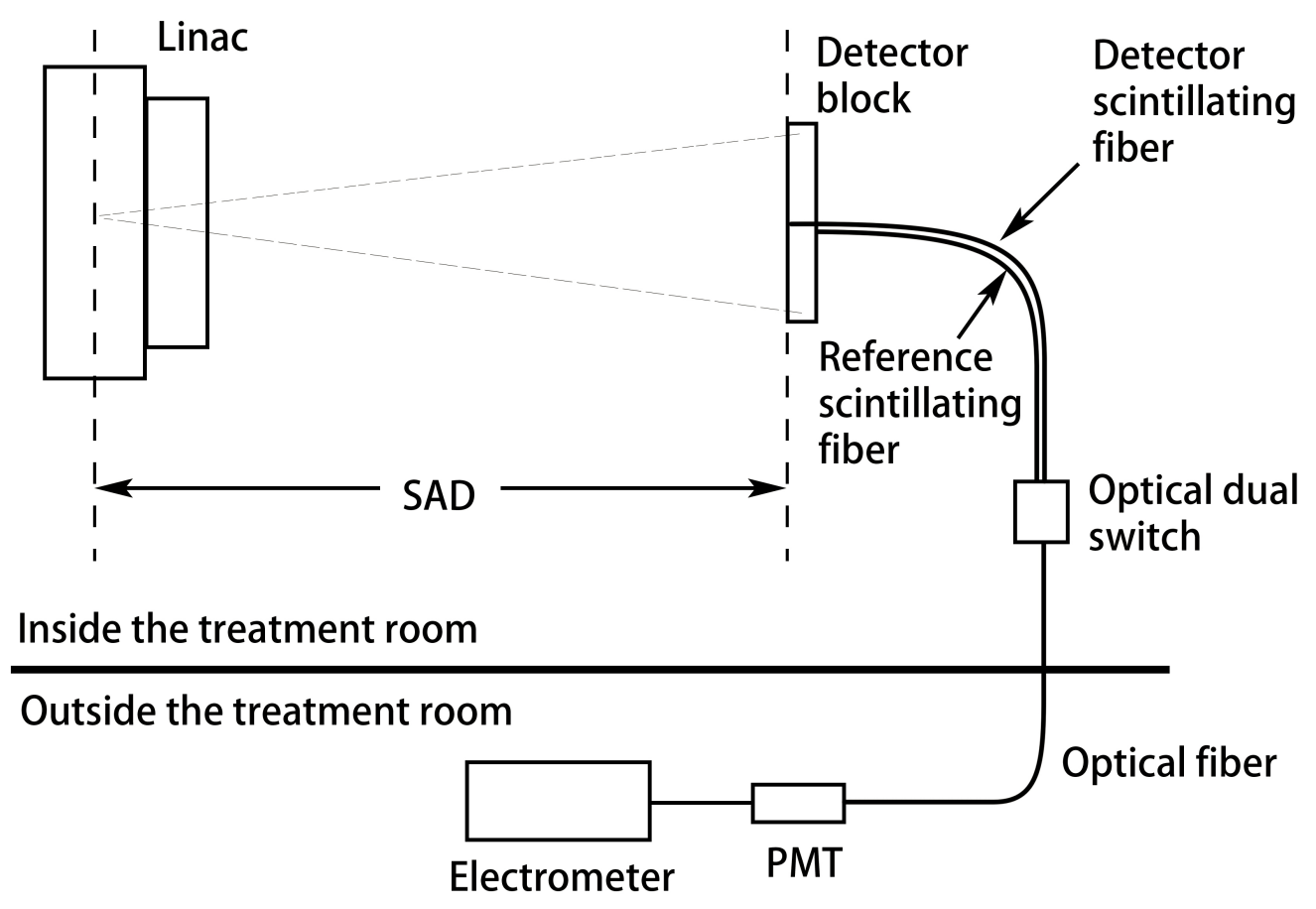

Figure 2.4: Experimental setup for linearity measurement of the detector. 
$\mu \mathrm{m}$ thickness in between. The slit assembly was placed on a rotation stage which was placed between the x-ray source and the detector (Fig. 2.5). The rotation stage can rotate at 1/300 $\%$ step.

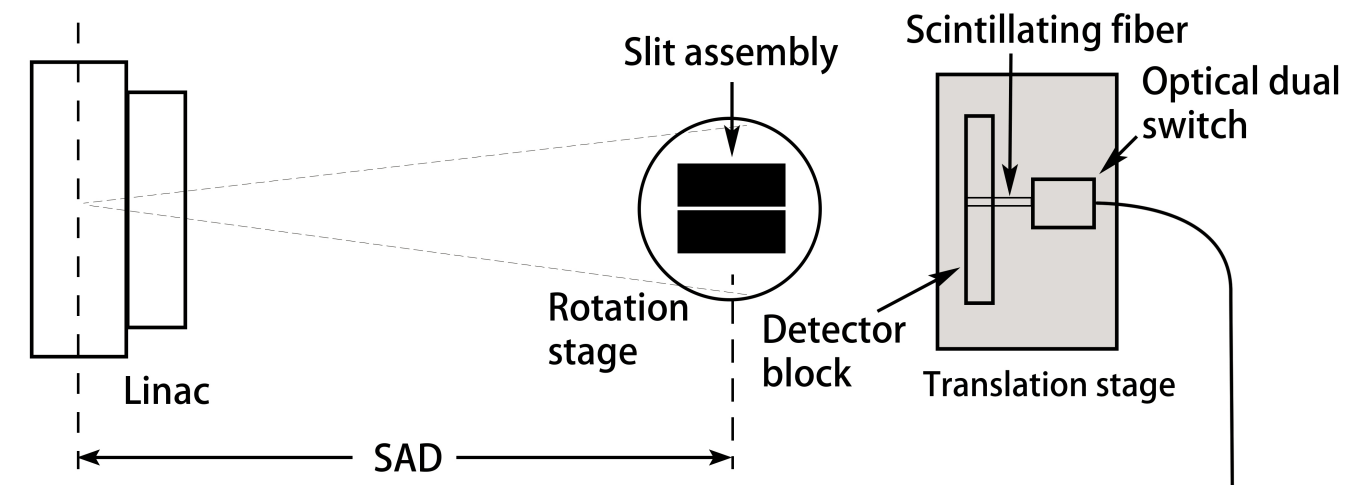

Inside the treatment room

Outside the treatment room

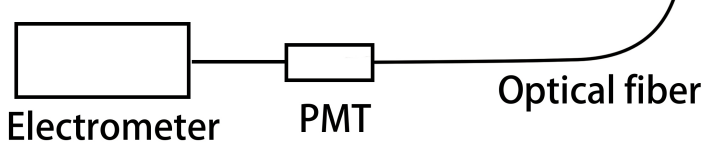

Figure 2.5: Experimental setup for line spread function measurement.

Before taking readings using the detector, an ionization chamber was used to align the slit assembly with the incident x-ray beam. To align the slit, ionization chamber readings were recorded for a given dose at different rotation stage angles. The alignment was achieved when ionization chamber reading was at the maximum (Fig. 2.6). The alignment was then checked with a radiochromic film attached to the beam exit surface of the slit. An exposure to 284 cGy produced a dark line on the radiochromic film.

The detector on a translation stage with a precision of $0.01 \mathrm{~mm} / \mathrm{step}$ was placed $50 \mathrm{~cm}$ away from the slit object to minimize the scattered radiation from the slit object. The radiation field was set to $3 \times 3 \mathrm{~cm}^{2}$ at the ISO to reduce the effect of scattered radiation. The prototype detector was then moved to scan across the slit beam (from -15 to $15 \mathrm{~mm}$ ) to obtain a raw LSF. To account for any residual signal from scattered radiation and leakage radiation through the $10.5 \mathrm{~cm}$ thick steel blocks, a "no-slit" scan was obtained when the slit was rotated $2^{\circ}$ off alignment. The difference between the "slit" data (i.e., the raw LSF data) and the "no-slit" data yielded the corrected LSF. 


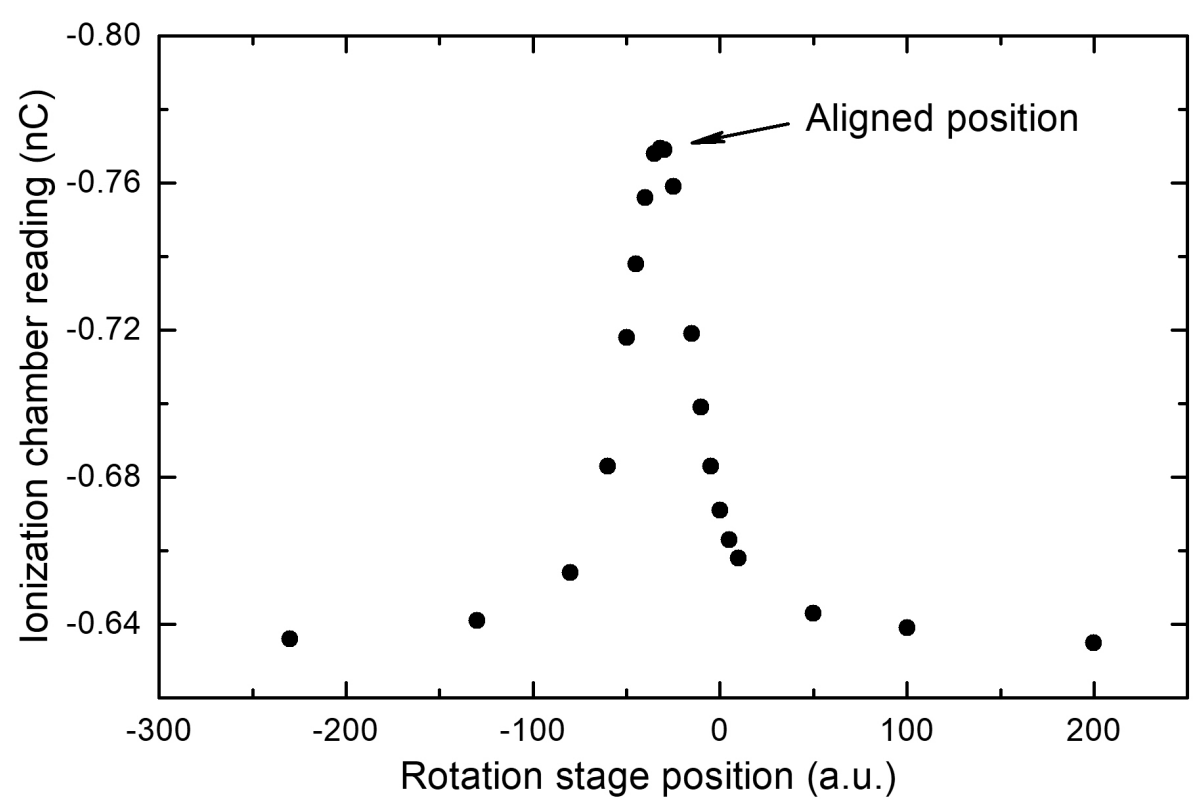

Figure 2.6: Ionization chamber reading as a function of rotation stage position. The peak location indicates that the slit object in Fig. 2.5 is aligned to the incident x-ray beam.

The corrected LSF was processed using the method in Ref. [51. The LSF was first made symmetric with respect to the center of the LSF and then the tail of the LSF was fitted analytically before applying the Fourier transform. The modulus of the Fourier transform yielded the measured MTF after normalized to unity at zero frequency. The measurements were repeated on different days to estimate measurement uncertainties.

\subsection{Anti-scatter property}

To measure the scatter to primary ratio (SPR) of the detector, a $30 \times 30 \times 30 \mathrm{~cm}^{3}$ solid water phantom was used to mimic the patient and generate scattered $x$ rays (Fig. 2.7). The center of the solid water phantom was placed at the ISO. The air gap (AG) which is the distance between the x-ray exit surface from the solid water and the detector was varied from $5 \mathrm{~cm}$ to 75 $\mathrm{cm}$. The light output from the scintillating fiber was measured using the PMT with a supply voltage of $500 \mathrm{~V}$ for a given dose.

The signal measured in Fig. 2.7 was generated from the fiber segment embedded in the detector plus the fiber portion that connects to the optical switch.

To get the signal only from the fiber segment in the detector, the fiber outside the detector was cut off and the end blacked (Fig. 2.8). The measurements were repeated. The signal 


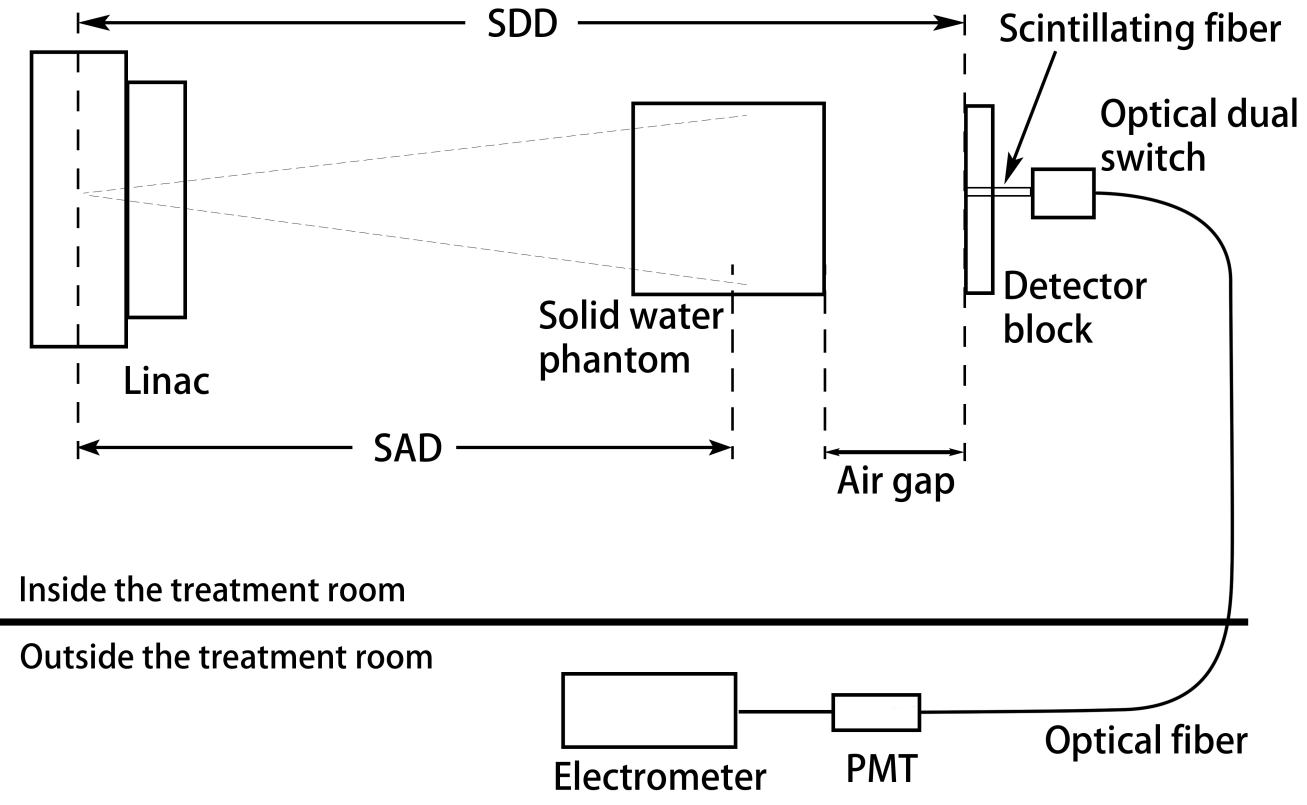

Figure 2.7: Experimental setup for measuring the anti-scatter properties of the detector.

difference from the two measurements yielded the signal from the fiber segment in the detector only. To calculate the SPR, the primary signal has to be determined. As the measured signal is always the sum of the primary signal and the scattered signal, the primary signal is obtained by extrapolating the measured signal to zero field size at each AG. When the field size approaches zero, the measured signal approaches the primary signal since at zero field size, scattered x-rays will not enter the detector. Primary signals were determined for measurements with and wihout the phantom placed between the x-ray source and the detector. Assuming that the total signal measured with solid water phantom at a given field size is $T$, then

$$
T=P+S,
$$

where $P$ and $S$ are the primary and scatter signals, respectively, in the presence of the phantom. Similarly, in the absence of the phantom, we have

$$
T^{\prime}=P^{\prime}+S^{\prime}
$$

where $T^{\prime}$ and $P^{\prime}$ are the total and primary signals without the phantom for the same Linac output, i.e., the same $\mathrm{MU}$ as that for the $T$, and $S^{\prime}$ is the scatter signal from the Linac 
collimators and the detector itself in the absence of the phantom.

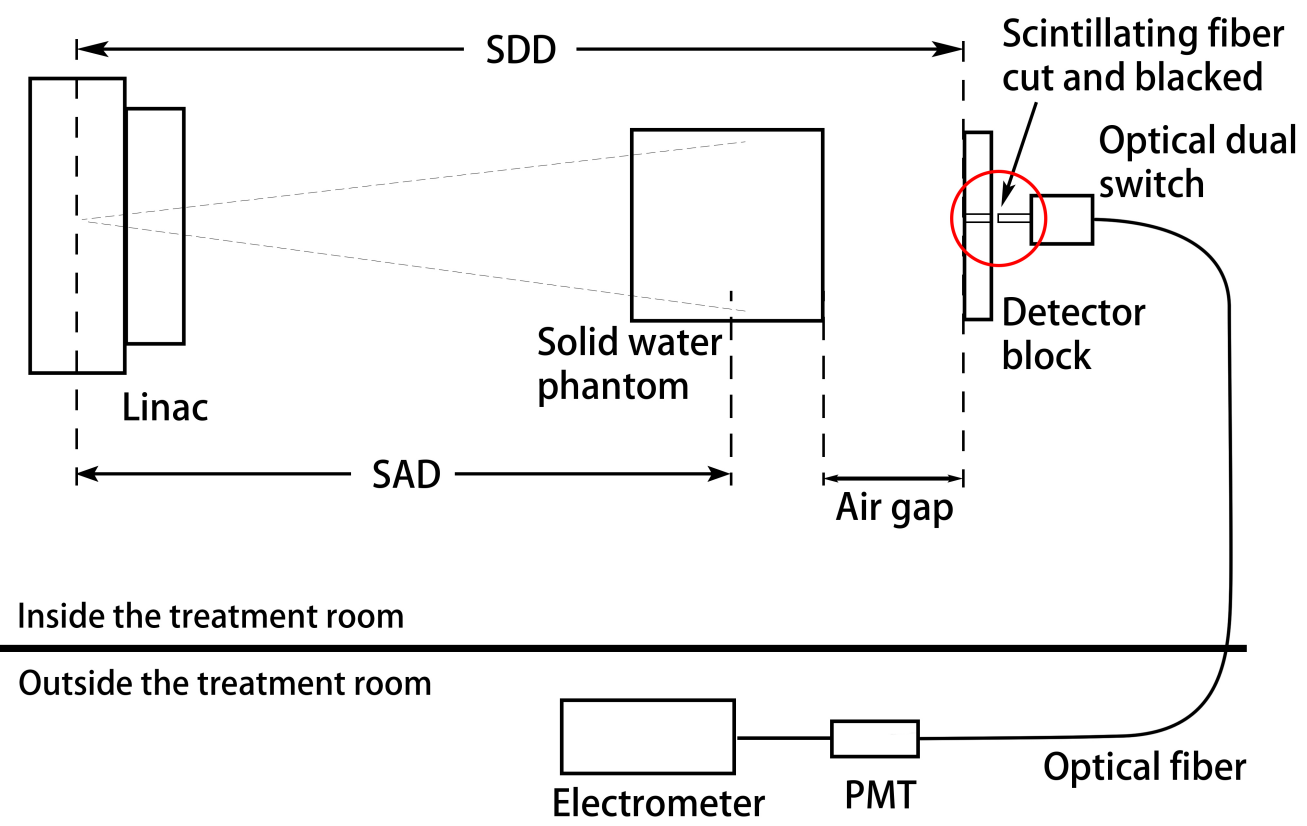

Figure 2.8: Experimental setup for measuring signal from the fiber segment outside the detector.

Figure 2.9 shows an example of data extrapolation to get the primary signal. To verify the extrapolated primary signal is correct, we investigated the dependence of $P$ and $P^{\prime}$ on source to detector distance $(S D D)$. Since high $\mathrm{QE}$ detectors are thick, the thickness of the detector should be considered. Therefore, we have 50

$$
P \propto \frac{1}{S D D(S D D+L)},
$$

where $L$ is the thickness $(2 \mathrm{~cm})$ of the detector. Furthermore we also calculated the ratio of $P$ to $P^{\prime}$ (denoted as $R_{0}$ ) and checked whether $R_{0}$ varies with air gap.

Once the primary signal $P$ is obtained, the $\mathrm{SPR}$ can be calculated from the measurement for a given field size

$$
\mathrm{SPR}=\frac{T-P}{P}
$$




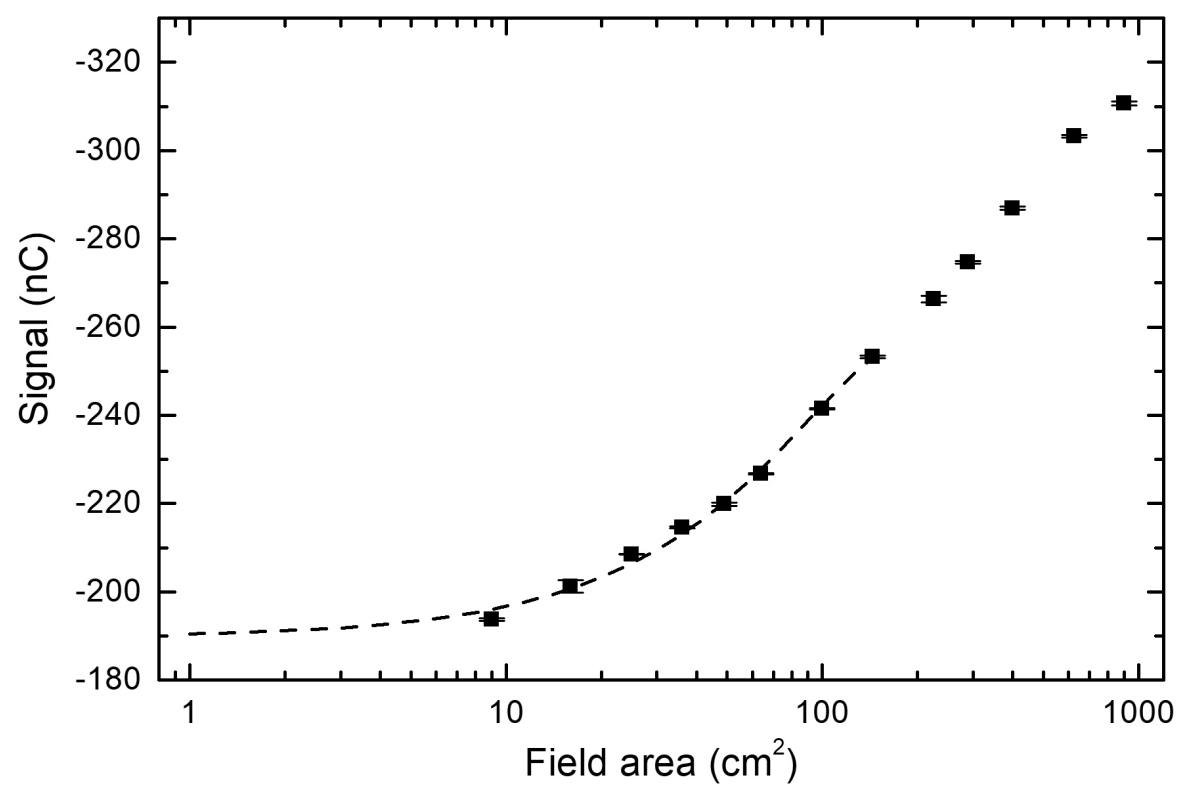

Figure 2.9: Detector signal as a function of field sizes for an air gap of $15 \mathrm{~cm}$. Dashed line is the extrapolation to field size zero.

\subsection{Sensitivity of the detector}

The best MV x-ray imaging system should be quantum-noise limited. To determine the sensitivity of the proposed detector, the signal and the root-mean-square (rms) noise per pixel was measured at a dose close to one Linac pulse. Since the Linac at Sunnybrook is not able to deliver a single Linac pulse (0.026 cGy) at a time, the slit object was used to attenuate the x-ray beam to reduce the dose to the detector. The experimental setup for sensitivity measurement is similar to the LSF measurement (Fig. 2.5). The slit object was first aligned to the incident beam and then rotated $4^{\circ}$ off alignment. The field size was set at $5 \times 5 \mathrm{~cm}^{2}$. Then $1 \mathrm{MU}$ was delivered and the electrometer reading recorded. The measurement was repeated 146 times. To determine the dose delivered after attenuated by the slit object, an ionization chamber was used to measure the dose with and without the slit object blocking the incident beam.

The signal $N_{p h}$ per pixel of the detector is defined as the number of scintillating photons exited in the effective region of the fiber, and can be calculated from the measured charge $Q$ of the PMT as 44

$$
N_{p h}=\frac{Q}{e \eta G \gamma \xi}
$$


where $e$ is the electron charge, $\eta$ is the average optical quantum efficiency of the PMT in the wavelength range from $420 \mathrm{~nm}$ to $560 \mathrm{~nm}$ (the emission band of the scintillating fiber, Section 3.1), $G$ is the gain of the PMT $\gamma$ is the light transmission coefficient through the optical dual switch and the optical fiber, and $\xi$ is the light transmission coefficient at the interface between the optical fiber and the PMT. $G$ was determined to be $2.41 \times 10^{5}$ at $750 \mathrm{~V}$ from the PMT specification provided by the manufacturer. $\gamma$ was introduced to account for the reflective light loss at the interface between the scintillating fiber and the optical dual switch and the light attenuation in the approximately $10 \mathrm{~m}$ long optical fiber. The value of $\gamma$ was measured to be 0.40 (Section 3.2). $\xi$ accounts for the reflective light loss at the interface between the optical fiber and the PMT. It was estimated that $\xi=0.85$ using the Fresnel equation at normal incidence 44 . The average optical QE of the PMT, $\eta$, was calculated using

$$
\eta=\int_{\lambda_{1}}^{\lambda_{2}} \eta(\lambda) \frac{\mathrm{d} N(\lambda)}{\mathrm{d} \lambda} \mathrm{d} \lambda / \int_{\lambda_{1}}^{\lambda_{2}} \frac{\mathrm{d} N(\lambda)}{\mathrm{d} \lambda} \mathrm{d} \lambda
$$

where $\lambda_{1}=420 \mathrm{~nm}$ and $\lambda_{2}=560 \mathrm{~nm}, \eta(\lambda)$ is the optical QE of the PMT at wavelength $\lambda$, and $N(\lambda)$ is the scintillating fiber emission spectrum measured in Section 3.1. The value of $\eta$ was calculated as 0.18 for $420 \mathrm{~nm}<\lambda<560 \mathrm{~nm}$.

From Eq. 2.5, we have the mean signal

$$
\left\langle N_{p h}\right\rangle=\frac{\langle Q\rangle}{e \eta G \gamma \xi},
$$

where $\langle\cdots\rangle$ means an average over large number of measurements.

The rms noise of the signal per pixel is defined as 44

$$
\sigma_{p h}=\sqrt{\left\langle N_{p h}^{2}\right\rangle-\left\langle N_{p h}\right\rangle^{2}}
$$

According to the theory of noise transfer through a stochastic gain process [52] and based on Eq. 2.5, we have

$$
\frac{\sigma_{d}^{2}}{e^{2}}=G^{2}\left[\eta^{\prime 2} \sigma_{p h}^{2}+\left\langle N_{p h}\right\rangle \eta^{\prime}\left(1-\eta^{\prime}\right)\right]+\eta\left\langle N_{p h}\right\rangle \sigma_{P M T}^{2}
$$

where $\eta^{\prime}(<1)$ and $\sigma_{d}$ are defined as

$$
\begin{gathered}
\eta^{\prime}=\eta \gamma \xi \\
\sigma_{d}=\sqrt{\left\langle Q^{2}\right\rangle-\langle Q\rangle^{2}} .
\end{gathered}
$$


The quantity $\sigma_{P M T}^{2}$ is the variance of the PMT gain and is given by 44

$$
\sigma_{P M T}^{2}=\frac{G^{2}}{\delta-1}
$$

where $\delta=3.09$ is the average gain per stage at $750 \mathrm{~V}$ which was the PMT voltage used for the sensitivity measurement.

Based on Eqs. 2.7, 2.9, 2.10, 2.11, and 2.12, we can calculate the mean signal $\left\langle N_{p h}\right\rangle$ and $\mathrm{rms}$ noise $\sigma_{p h}$ of the proposed detector. In the above noise analysis, the Linac output noise was neglected since it is small comparing to the PMT noise, accounting for only 1-2\% of the total noise 44. 


\section{Chapter 3}

\section{Results and discussion}

\subsection{Scintillating fiber emission spectrum}

Figure 3.1 shows the scintillating fiber emission spectrum in absolute irradiance excited by a $6 \mathrm{MV}$ beam at the gantry angle 0 . Red line is the average of 78 measurements (black lines). The emission peak is at $450 \mathrm{~nm}$, which is in the most sensitive region of the PMT used (Fig. 3.2 . Since each of the measured spectrum shown in Fig. 3.1 is the spectrum measured in a $6 \mathrm{MV}$ beam minus the background, there are negative values in the background region of the spectrum, i.e., outside the fiber emission band. The negative values are not shown in Fig. 3.1. The average of many measurements in the wavelength range outside the fiber emission band should be 0 , which is indicated by the red line.

Figure 3.3 shows the fiber emission spectra at different gantry angles. The high energy edge (shorter wavelength) and the peak position of the emission spectrum are the same for excitation at different gantry angles. The low energy emission tail is smaller as the gantry angle increases. This is due to the fact that the incident beam has to go through a larger solid water depth to excite the fiber and the low energy component of the $6 \mathrm{MV}$ beam is attenuated more as the gantry is tilted to a larger angle. As the x-ray beam gets harder, the low energy tail of the emission spectrum gets smaller.

Figure 3.4 shows the fiber emission spectra excited by x-ray beams of different energies. The high energy edge and peak position are the same for the emission spectra excited by beams with different qualities. The low energy tail of the spectra excited by $\mathrm{kV}$ beams are higher than that excited by the $6 \mathrm{MV}$ beam, which means when the x-ray beam is softer, the low energy tail is larger. This is in agreement with what Fig. 3.3 shows. 


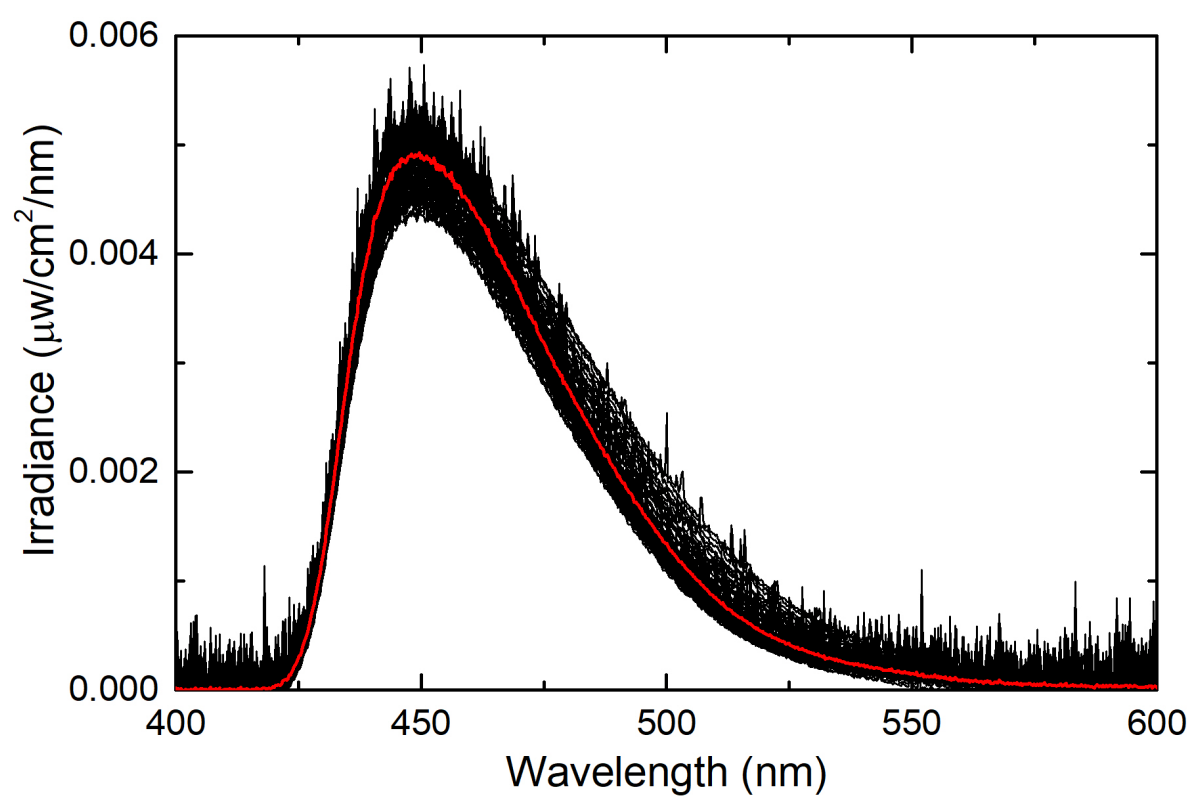

Figure 3.1: Scintillating fiber emission spectrum excited by a 6 MV beam. Red line is the average of 78 measurements (black lines).

\subsection{Signal attenuation due to the optical dual switch and opti- cal fiber}

Figure 3.5 shows the tungsten halogen light source emission spectra measured using setup shown in Fig. 2.3. The troughs are due to absorption by the scintillating fiber. As can be seen, the signal is attenuated significantly by the optical switch and the optical fiber. The ratio of the absolute irradiance from measurement 2 to that from measurement 1 is the ratio of signal transmitted through the optical switch and optical fiber. The ratio of the signal transmitted in the wavelength range from $450 \mathrm{~nm}$ (the emission peak of the scintillating fiber) to $560 \mathrm{~nm}$ (the upper wavelength limit of the scintillating fiber emission spectrum) is plotted in Fig. 3.6. The transmission ratio in the fiber emission band is about 0.40 .

\subsection{Linearity}

Figure 3.7 shows the measured signal from the fiber segment in the detector block as a function of dose at the field size $2.4 \times 2.4 \mathrm{~cm}^{2}$. The solid line is the linear fit to those data points. The measurement uncertainty is smaller than the size of the data points. The signal is indeed linear in the dose range measured. 


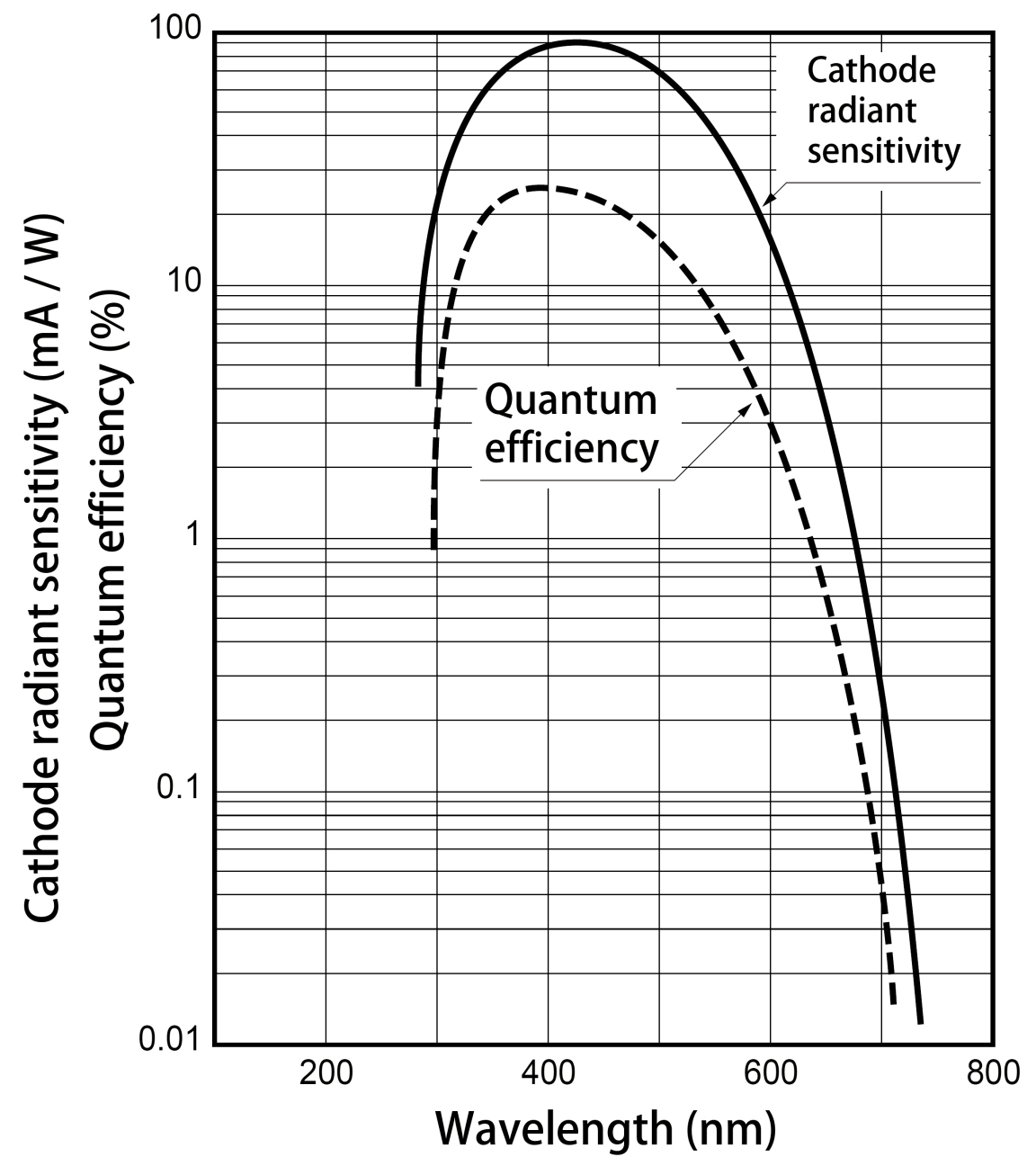

Figure 3.2: Cathode radiant sensitivity and quantum efficiency of the PMT used in this work as a function of wavelength (Source: manufacturer product data sheet). 


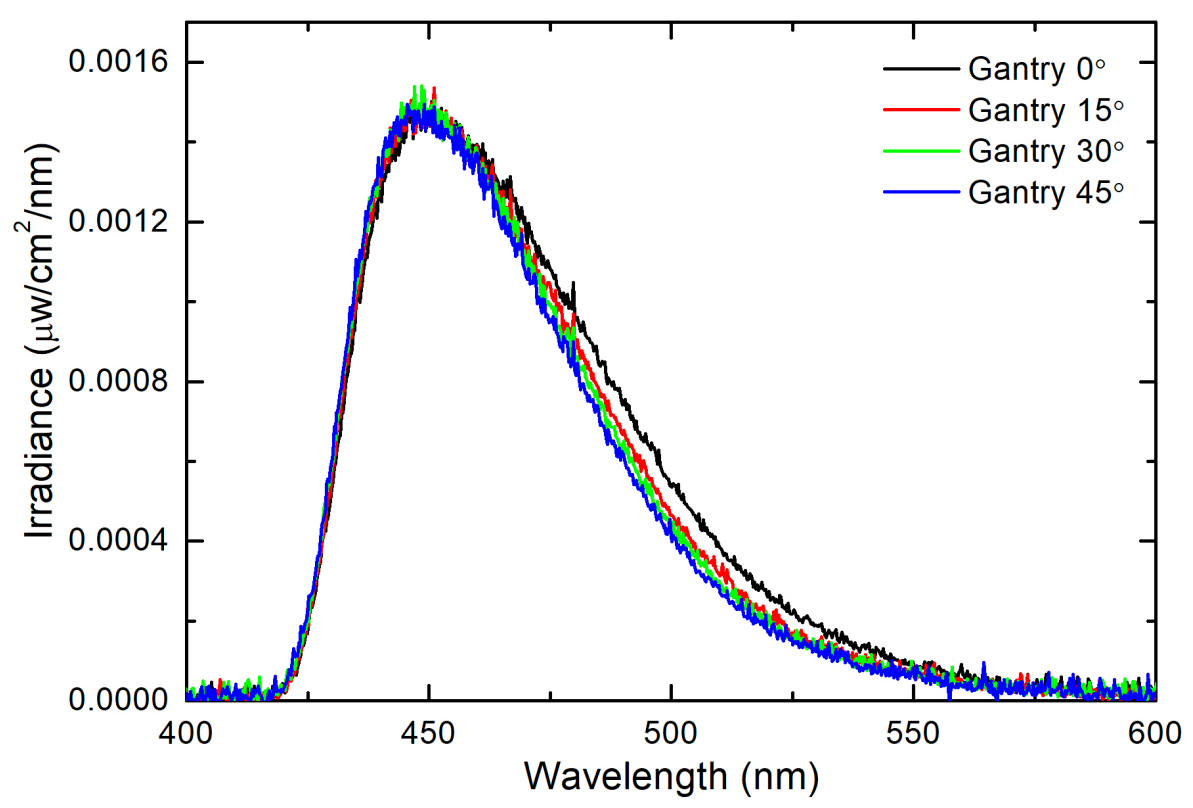

Figure 3.3: Scintillating fiber emission spectra excited by a $6 \mathrm{MV}$ beam at different gantry angles.

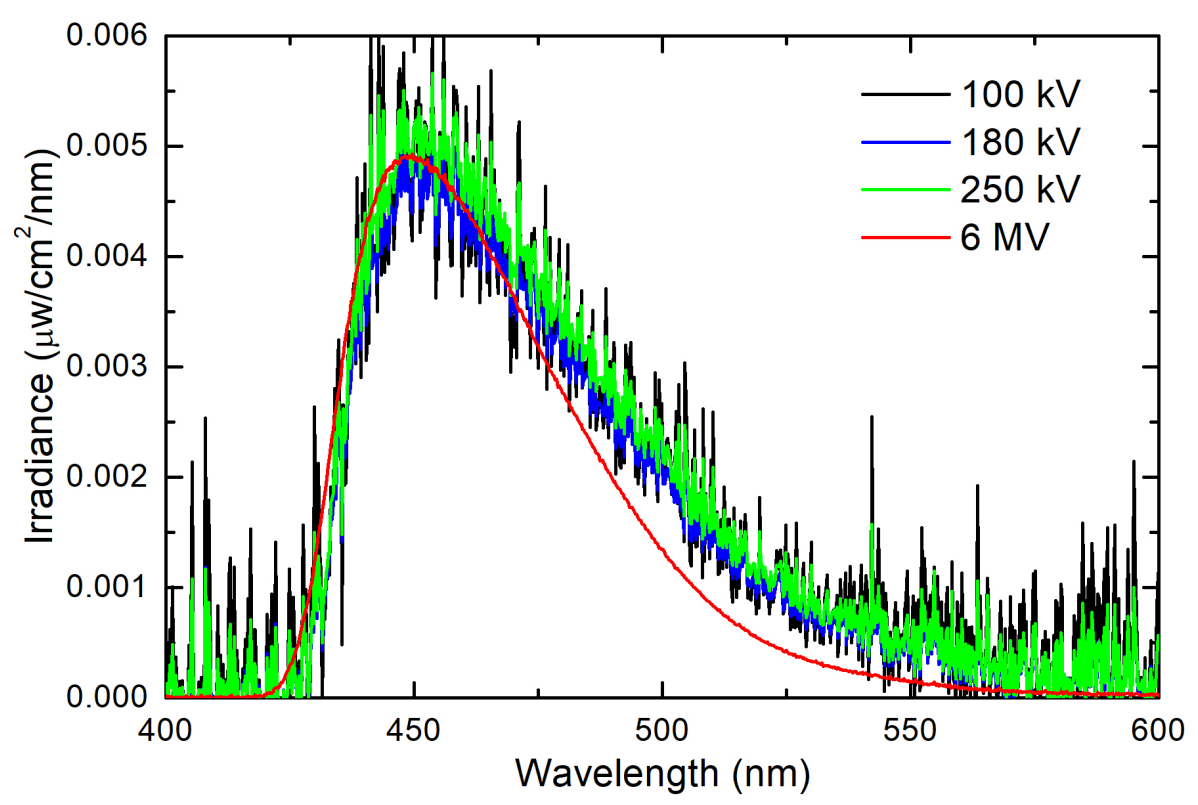

Figure 3.4: Scintillating fiber emission spectra excited by kV and MV beams. 


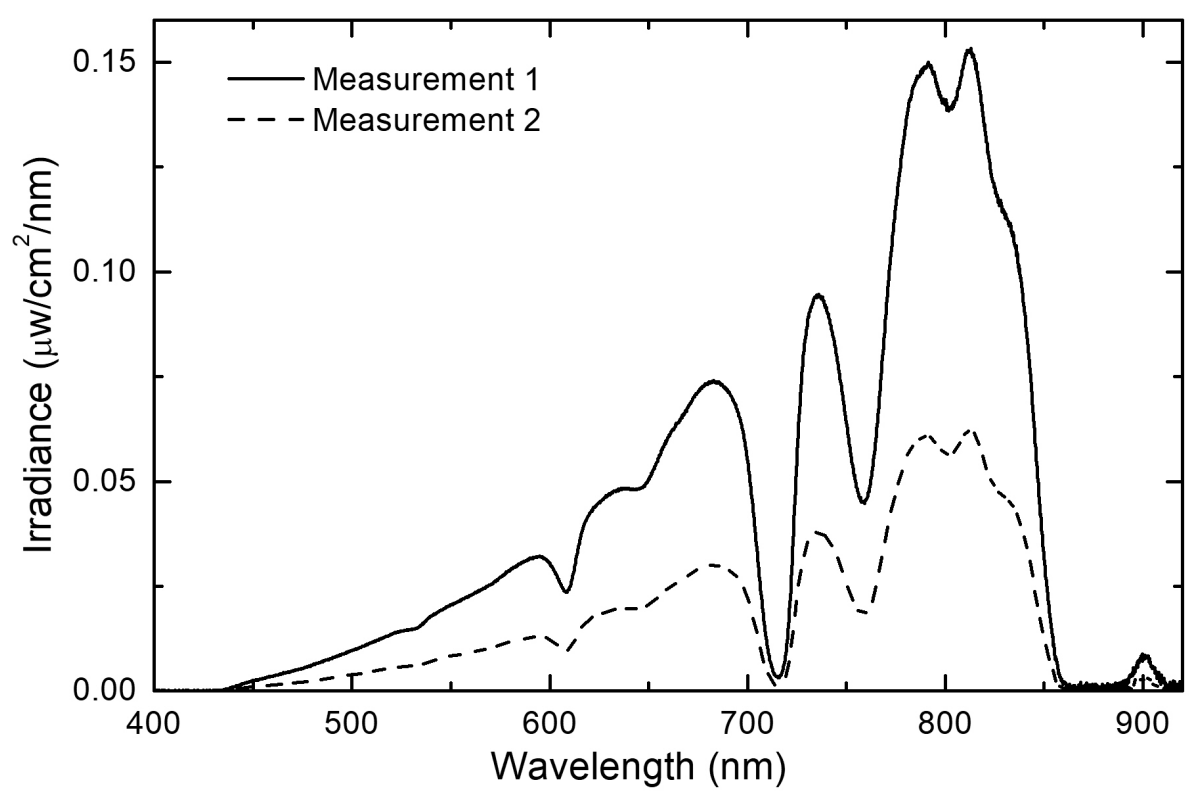

Figure 3.5: Lamp emission spectrum measured using the setup shown in Fig. 2.3 ,

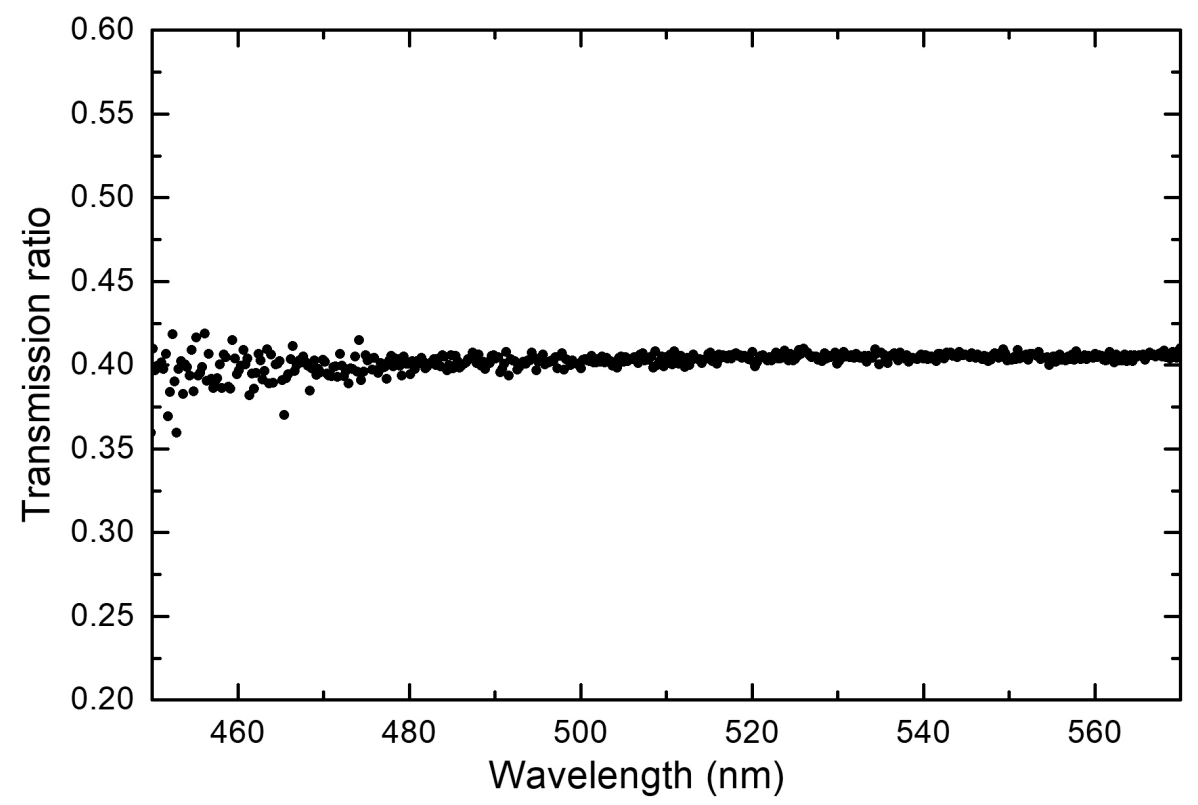

Figure 3.6: Transmission ratio of the light intensity through the optical switch and the optical fiber in the wavelength range from $450 \mathrm{~nm}$ to $560 \mathrm{~nm}$. 


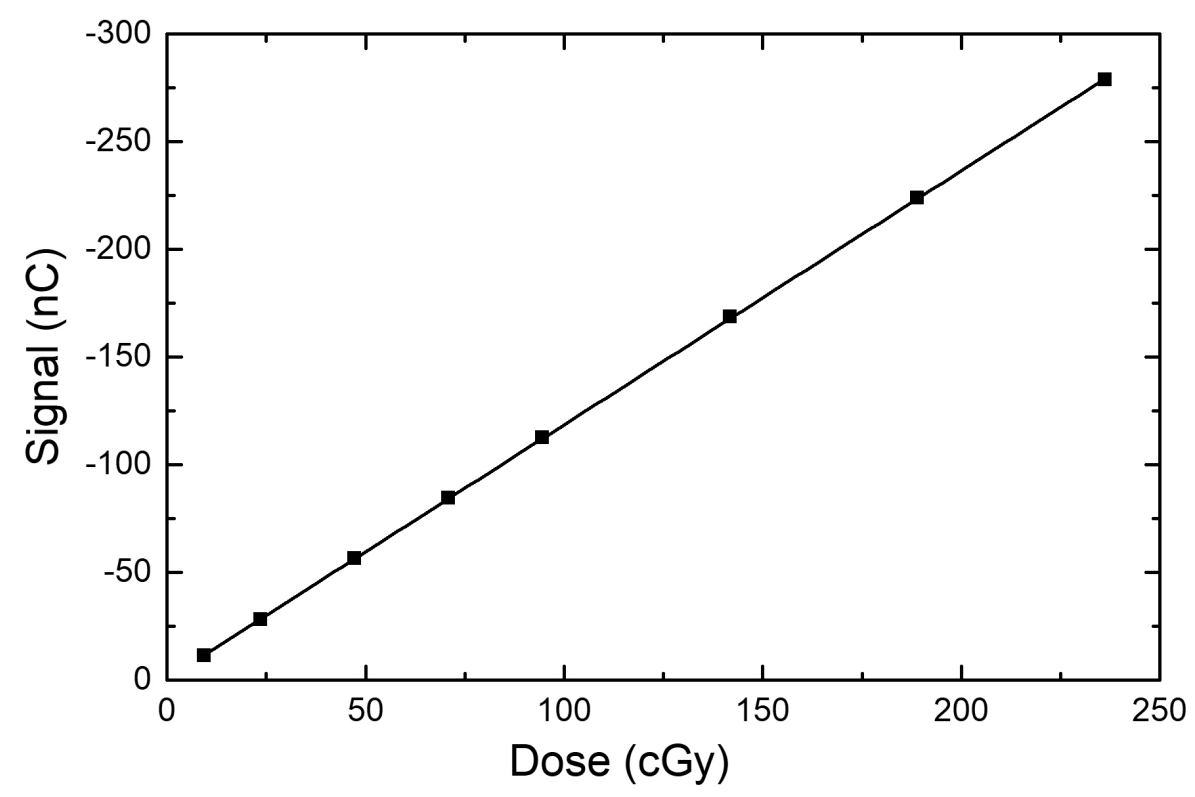

Figure 3.7: Detector signal measured by the photomultiplier tube as a function of incident dose.

\subsection{Quantum efficiency}

The measured QE was $35 \%$ at $6 \mathrm{MV}$ beam and independent of the $\mathrm{x}$-ray field size. The QE was also calculated using the mass attenuation coefficient database from the National Institute of Standards and Technology, Gaithersburg, MD [53 and the detector composition shown in Fig. 2.1. The BC-600 optical epoxy is made of $78 \%$ bisphenol A diglycidyl ether resin and $22 \%$ diethylene glycol, di(3-aminopropyl) ether, and has a density of $1.18 \mathrm{~g} / \mathrm{cm}^{3}$. The calculated QE is $35.8 \%$. The slight difference between the measured and calculated $\mathrm{QE}$ is probably due to the energy spectrum of the $6 \mathrm{MV}$ beam used in the calculation. Synergy-6X beam spectrum (Fig. 1.5 with discrete energies was used in the calculation, while the actual $6 \mathrm{MV}$ beam is a continuous spectrum.

\subsection{Spatial resolution}

Figure 3.8 shows the LSF $\$$ of the prototype detector in the $\mathrm{x}$ and $\mathrm{y}$ directions (denoted in Fig. 2.1. The full width at the half maximum of the LSF is $\sim 1.4 \mathrm{~mm}$. The LSF signal measured in the $\mathrm{y}$ direction is slightly higher than that measured in the $\mathrm{x}$ direction. The LSF data were made symmetric before doing Fourier transform to obtain the MTFs following Ref. [51]. Figure 3.9 shows the MTF in the $\mathrm{x}$ and $\mathrm{y}$ directions. The measurement uncertainty was estimated 
based on two measurements on different days. The frequency at $50 \%$ modulation in y direction is $0.2 \mathrm{~mm}^{-1}$, which is comparable to the video based EPIDs, but lower than the current flatpanel EPIDs with a $f_{50}$ of about $0.3-0.4 \mathrm{~mm}^{-1}[26,54$. The spatial resolution in the $\mathrm{x}$ direction is not as high as that in the y direction.

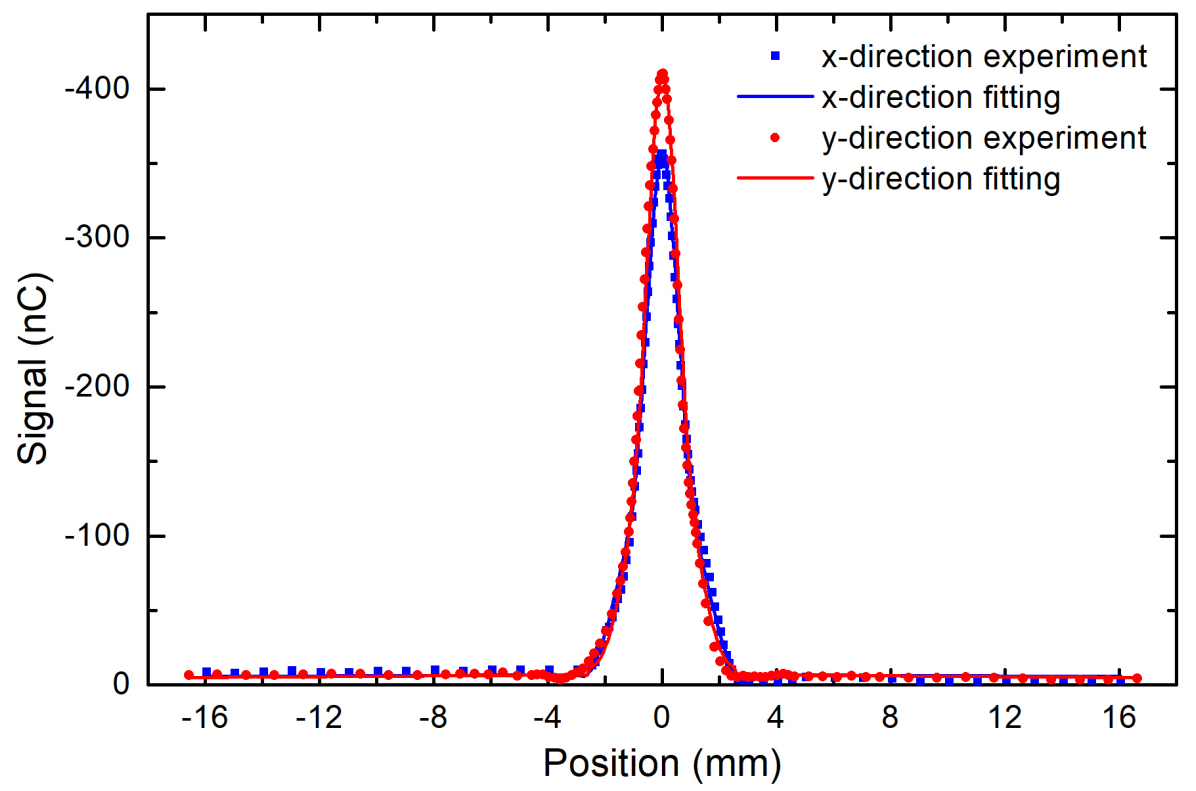

Figure 3.8: Line spread function of the detector in the $\mathrm{x}$ and $\mathrm{y}$ directions.

\subsection{Anti-scatter property}

The primary signal of the detector at different AGs were obtained by extrapolating the signal to field size zero, as shown in Fig. 2.9. At each AG, the field size was varied between $3 \times 3$ and $30 \times 30 \mathrm{~cm}^{2}$ and at each field size, the measurement was repeated three times. Figure 3.10 shows the primary signals with and without phantom follow the inverse square law well, as indicated by the curve fitting results. Ratios of the primary signals in the presence of the phantom to those without the phantom, $R_{0}$, at different AGs are listed in Table 3.1. $R_{0}$ does not change with the AG in the range from $15 \mathrm{~cm}$ to $75 \mathrm{~cm}$, which is expected, and it has a value of about 0.61 . The $R_{0}$ at $5 \mathrm{~cm} \mathrm{AG}$ is 0.65 , higher than that at other AGs. This is probably due to that at $5 \mathrm{~cm}$ AG in the presence of the phantom, the secondary electrons produced in the phantom are able to travel to the detector and excite optical photons in the scintillating fiber.

Figure 3.11 shows the SPR as a function of field size at different AGs. The results from 


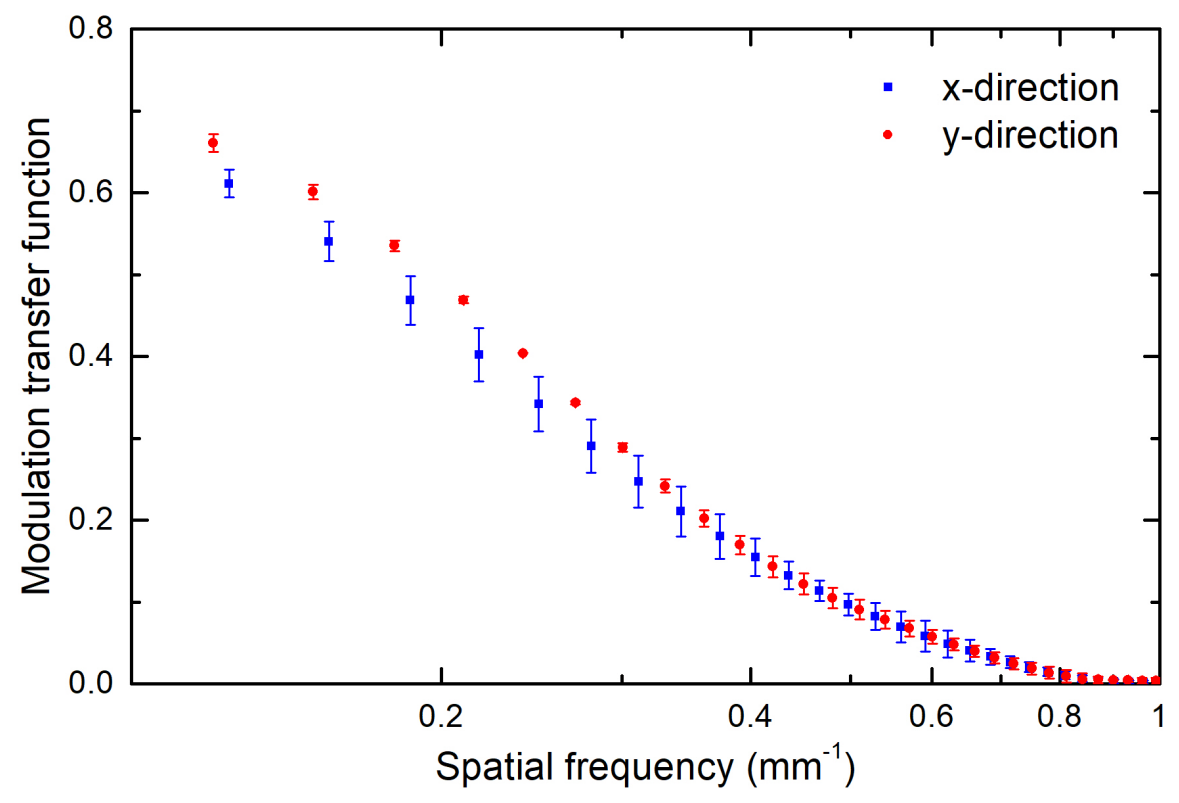

Figure 3.9: Modulation transfer function of the detector in the $\mathrm{x}$ and $\mathrm{y}$ directions.

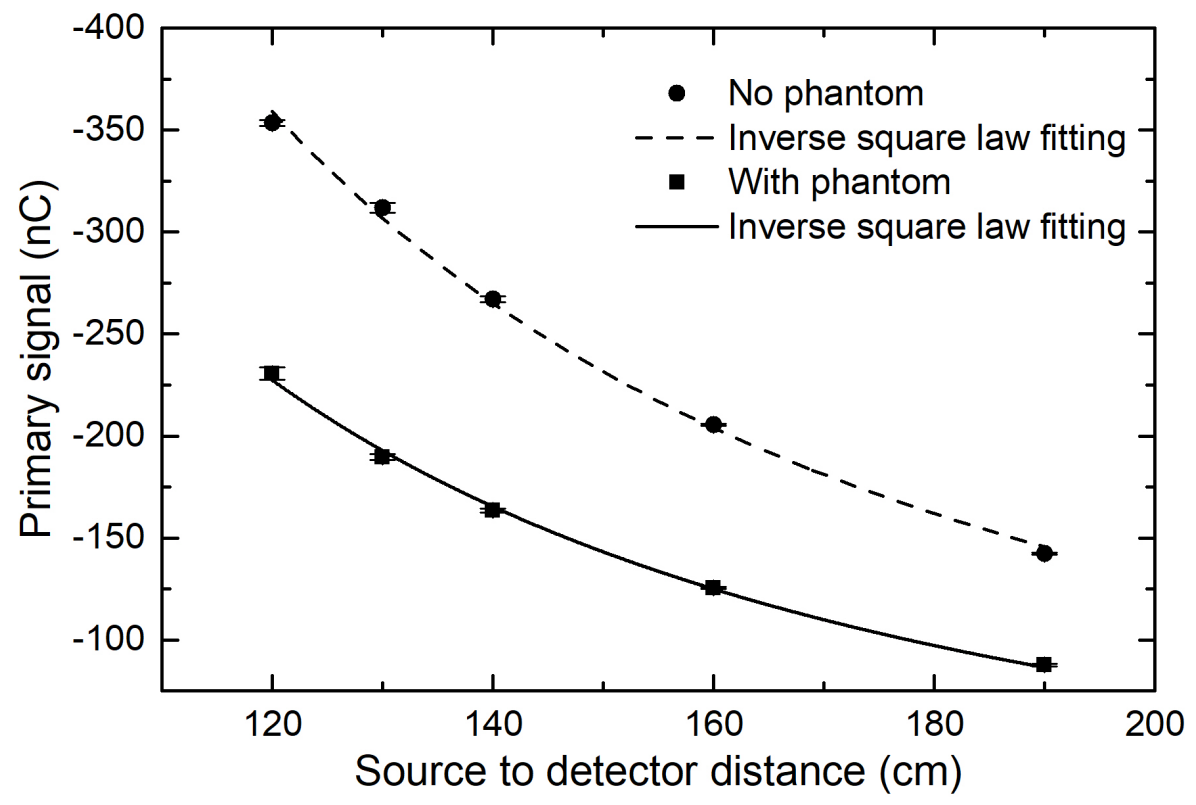

Figure 3.10: Detector signals due to the primary beam with and without phantom as a function of source to detector distance. 
Table 3.1: Ratios $\left(R_{0}\right)$ of the primary signals in the presence of the phantom to those without the phantom at different air gaps.

\begin{tabular}{cc}
\hline \hline Air gap $(\mathrm{cm})$ & $R_{0}$ \\
\hline 5 & 0.652 \\
15 & 0.608 \\
25 & 0.612 \\
45 & 0.610 \\
75 & 0.617 \\
\hline \hline
\end{tabular}

the scintillating fiber based detector are compared to that based on the ionization chamber published earlier (Ref. [50]). The SPR decreases with the increase of the AG and increases with the field size for both types of detectors. For the same AG and field size, the SPR of the scintillating fiber detector is always lower than that of the ionization chamber detector. For the scintillating fiber detector, the $\mathrm{SPR}$ at $10 \mathrm{~cm}$ AG should be between the $\mathrm{SPR} s$ at $5 \mathrm{~cm}$ and 15 $\mathrm{cm}$. At $10 \mathrm{~cm} \mathrm{AG} \mathrm{and} 400 \mathrm{~cm}^{2}$ field size, the SPR of the scintillating fiber detector is about $30 \%$ lower than that of the ionization chamber detector.

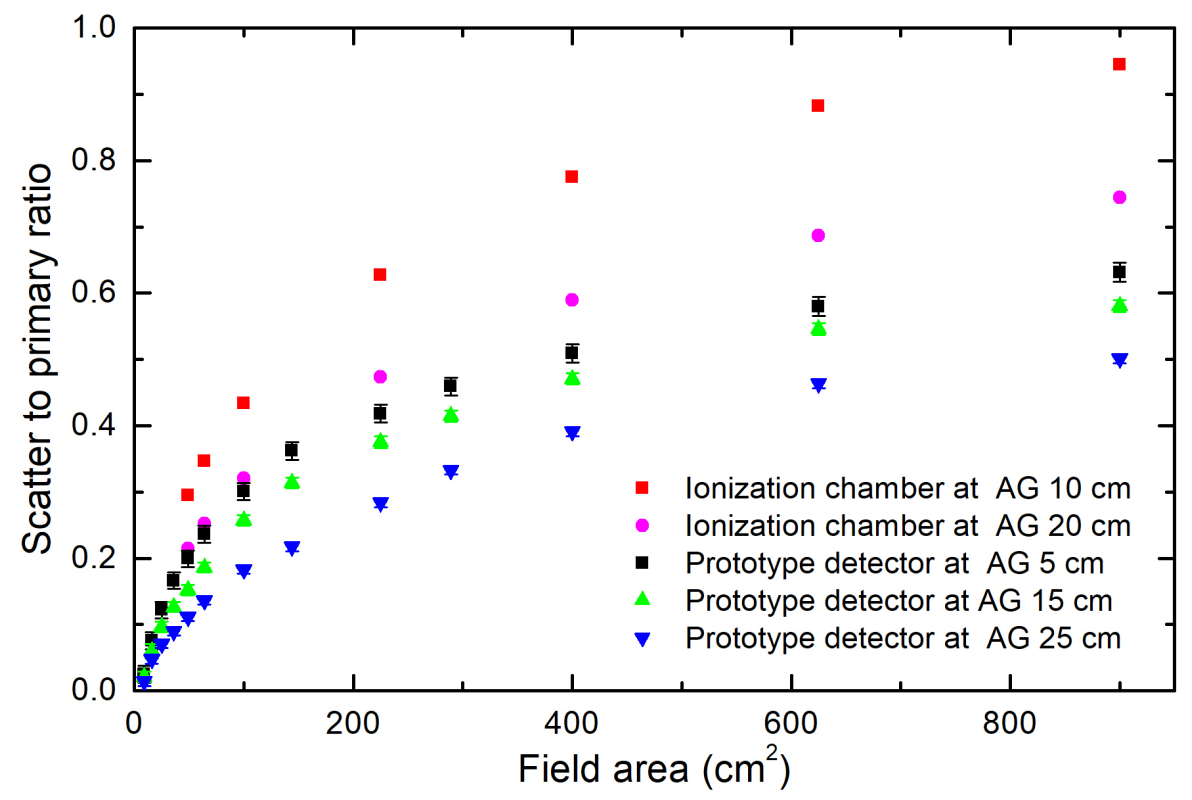

Figure 3.11: Scatter to primary ratios as a function of the field size at different air gaps for the prototype detector and ionization chamber detector. 


\subsection{Sensitivity of the detector}

Data acquired from sensitivity measurements are listed in Appendix 1. All charges measured are negative. Figure 3.12 shows the data distribution. The average value is $8.31 \mathrm{nC}$. It was determined that $95.8 \%$ of the incident beam was attenuated by the slit object. Considering the field size used for the measurement, the dose delivered per MU Linac output was 0.041 cGy to water at the ISO, which is less than two Linac pulses.

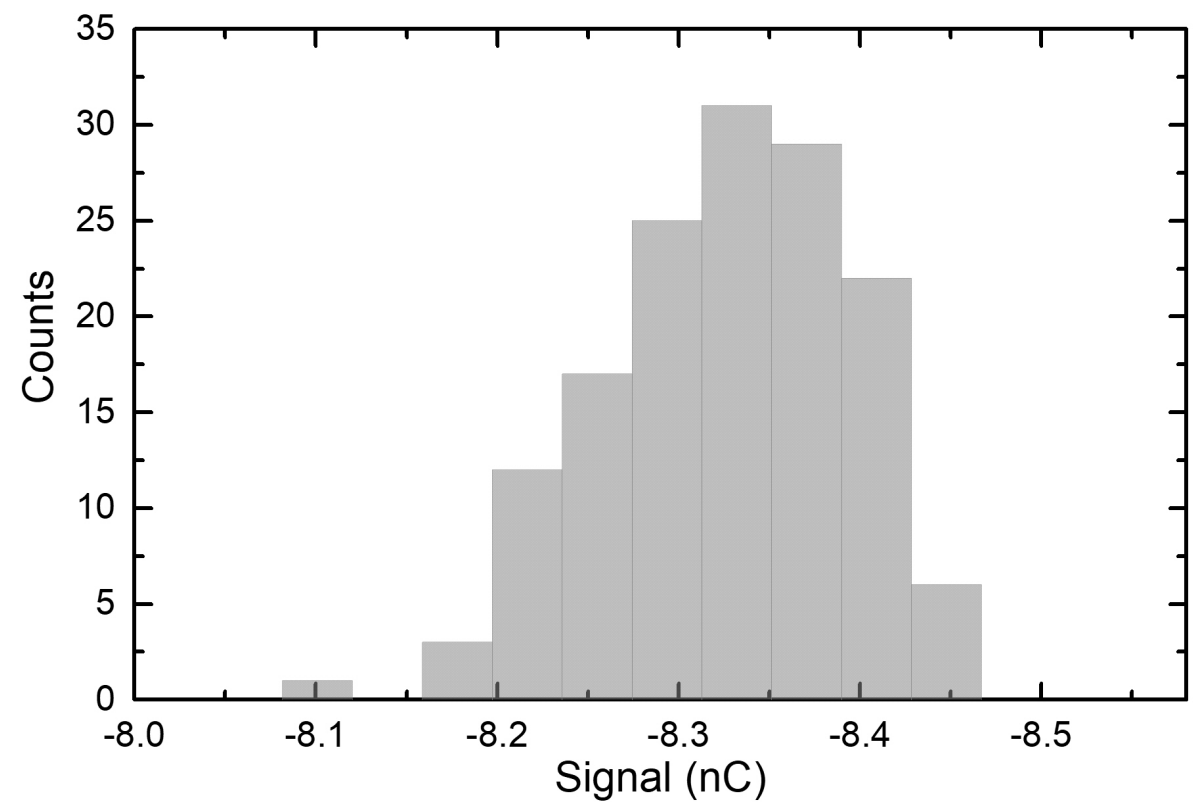

Figure 3.12: PMT reading distribution for detector sensitivity measurement.

The $\left\langle N_{p h}\right\rangle$ can be calculated as $3.52 \times 10^{6}$ and $\sigma_{p h} 2.69 \times 10^{4}$. The rms noise $\sigma_{p h}$, which includes the x-ray quantum and absorption noises at the dose $0.041 \mathrm{cGy}$, is much larger than the electronic noise per pixel (typically 2000e) of a conventional flat-panel imager [55]. Thus the prototype detector is quantum-noise limited.

\subsection{Discussion}

The $\mathrm{QE}$ in this work was defined as the percentage of $\mathrm{x}$ rays that interact with the detector. Some of the $\mathrm{x}$ rays that interact with the detector may not generate optical signals in the scintillation fiber. The range of the Compton electrons generated by $6 \mathrm{MV}$ beam in lead is about $1.4 \mathrm{~mm}$. The minimum thickness of the lead separation between the fibers is $0.25 \mathrm{~mm}$. As most of the Compton electrons are scattered forward, a significant number of secondary 
electrons generated in lead will not enter the fiber to produce optical signals. However, it is very difficult, if not impossible, to measure the ratio of the $\mathrm{x}$ rays that produce at least one optical photon detected by the PMT.

When measuring the LSF the signal in the y direction is slightly higher than that in the $\mathrm{x}$ direction (Fig. 3.8). This is due to the optical epoxy used to glue the lead and fibers. The BC-600 optical epoxy is highly transparent. The transmission through a $0.125 \mathrm{~mm}$ thick layer is greater than $98 \%$ for wavelength above $400 \mathrm{~nm}$ according to the manufacturer. Since in the $\mathrm{x}$ direction there is a channel of epoxy $(0.20 \mathrm{~mm}$ long $)$ connecting the fibers, there are cross-talks between fibers in the $\mathrm{x}$ direction when the slit object is oriented in the $\mathrm{x}$ direction to scan the detector in the y direction. Therefore, the LSF signal in the y direction is higher than that in the $\mathrm{x}$ direction. The cross-talk between fibers in the $\mathrm{x}$ direction also explains the lower spatial resolution in $\mathrm{x}$ direction comparing to that in the y direction, as shown in Fig. 3.9.

The spatial resolution of the prototype detector is not as good as the current flat-panel based EPID 5 [51] and can be improved. It has been shown that the spatial resolution of a thick and high QE detector is determined by both the range of energetic electrons produced by $\mathrm{x}$ rays interacting with the detector and the reabsorption of $\mathrm{x}$ rays scattered within the detector. The large diameter and low density of the fiber deteriorate the resolution though the spacing lead helps shorten the range of the secondary electrons. Using fibers with smaller diameter should improve the spatial resolution.

It is well understood that scattered $\mathrm{x}$ rays are detrimental to the image quality of an $\mathrm{x}$-ray imaging system. In general, there are three sources that produce scattered $\mathrm{x}$ rays in MV x-ray imaging in radiotherapy: Linac head, patient (phantom), and detector itself. Scattered signals from all three sources were included in the measured SPR in this study. Our results show that the prototype detector can suppress the scattered signal. This is due to the application of lead as the spacing material. Lead not only helps improve the absorption of primary x rays and thus increase the QE of the detector, but also acts like anti-scatter grid to some degree by blocking scattered $\mathrm{x}$ rays from producing signals in scintillating fibers.

The number of incident $\mathrm{x}$ rays, $N_{\mathrm{x}}$, per $\mathrm{MU}$ attenuated by the slit object was calculated using the fluence-to-dose conversion factor, $8.4 \times 10^{-8} \mathrm{~mm}^{2} \mathrm{cGy} /$ photon for the $6 \mathrm{MV}$ beam of our Linac 43, 56. $N_{\mathrm{x}}$ is $1.69 \times 10^{5}$ per pixel for a output dose of 0.041 cGy to water at the ISO. Therefore the number of detected optical photons generated per incident $\mathrm{x}$ ray is about 21. Since many coefficients were involved in calculating this number and some of the coefficients are from the product specification published by the manufacturer and not verified by experiments, the uncertainty associated with the calculation result was estimated to be $10-20 \%$.

The number of detected optical photons generated per incident $\mathrm{x}$ ray can be improved. The efficiency of detecting optical photons is not maximized for this prototype detector. Only half 
of the optical photons emitted in the scintillating fiber will propagate towards the $\overline{P M T}$ of which only those incident on the fiber wall at an angle equal to or greater than the critical angle that satisfies the total internal reflection condition can be detected by the $\mathrm{PMT}$. For the scintillating fiber used, using the data provided in Table 2.1, it can be calculated that only those photons with a incident angle equal to or greater than $72.4^{\circ}$ at the interface between the inner and outer claddings will be able to exit the fiber (Fig. 3.13), which accounts for only $5.3 \%$ of the total optical photons emitted in the fiber. Of these $5.3 \%$ photons, some will be lost due to the attenuation in the optical fiber and reflection at the interfaces. It would greatly increase the signal if the fiber wall and the surface facing the x-ray source are coated with a reflective layer. As the light yield in the scintillating fiber is high, we should be able to use smaller size fibers, e.g. fibers with a diameter of $0.5 \mathrm{~mm}$, to improve the spatial resolution and the detector is still quantum-noise limited. The cross talk between fibers in the $\mathrm{x}$ direction will also be eliminated by coating the fibers with a reflective layer.

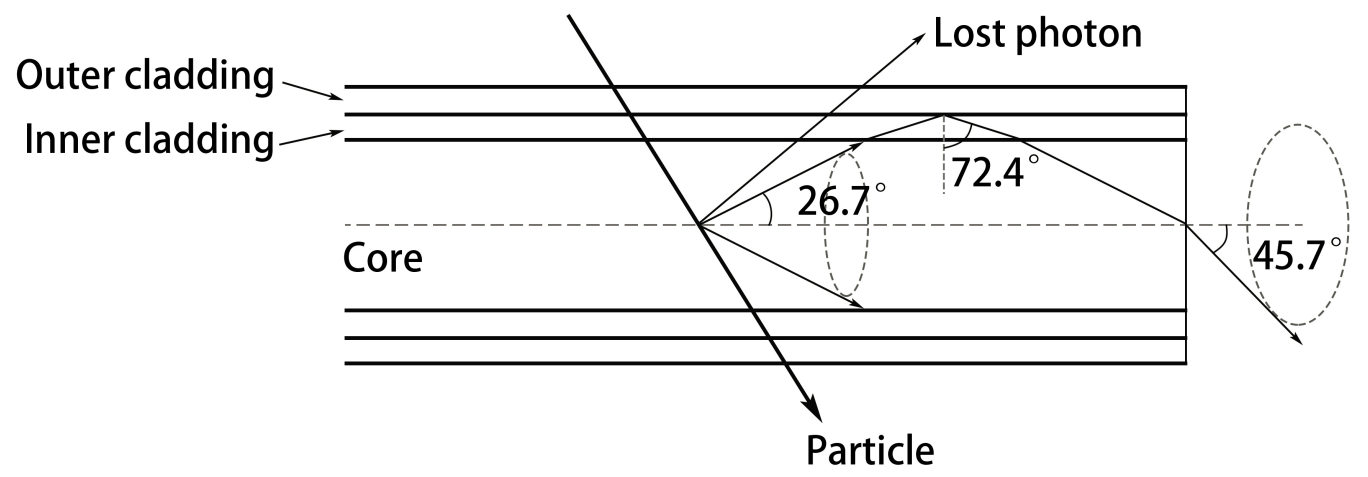

Figure 3.13: Diagram showing the optical photons that are excited in the scintillating fiber and able to exit the fiber. 


\section{Chapter 4}

\section{Conclusion and future work}

\subsection{Conclusion}

Properties of a new high QE MV x-ray detector for IGRT applications were experimentally evaluated. This detector responds linearly to the incident radiation dose. It has a $\mathrm{QE}$ of $35 \%$ at $2 \mathrm{~cm}$ thickness, which is about an order of magnitude higher than that of current EPIDs. The resolution of the prototype detector is comparable to the video based EPIDs, but lower than the flat-panel based ones. The resolution can be improved by optimization of the design parameters. Due to the application of lead as the septal material between scintillating fibers, the new detector also has anti-scatter property, which will help improve the SNR of the image. As scintillating light yield is high, this detector is quantum-noise limited at a very low dose, i.e., a couple of Linac pulses. Further investigation to improve the detector performance is warranted.

This work is the first experimental study of this type of detector for radiotherapy applications. The success of this project will result in a new treatment verification tool in the treatment room of radiotherapy and new knowledge in the application of plastic scintillating fibers in portal imaging.

\subsection{Future work}

The frequency-dependent NPS and DQE were not measured in this study since the pixel-topixel noise correlation cannot be determined from measuring signals from only one fiber. The next step is to couple a 2D detector to a flat-panel imager and measure the NPS and DQE.

We are currently doing Monte Carlo simulation of the properties of this prototype detector. Once simulation results are validated, design parameters of the detector, such as the fiber 
diameter, lead spacing thickness and detector thickness, can be optimized using the simulation to achieve a better combination of the $\mathrm{QE}$ and spatial resolution of the detector.

Once the prototype detector is optimized for the QE and spatial resolution and coupled to a flat-panel imager, we can use it to image phantoms to evaluate its performance in situations similar to clinical cases. The application of this detector in the MV CBCT should, in particular, be explored, which will produce $3 \mathrm{D}$ MV verification images instead of the $2 \mathrm{D}$ projections with clinically acceptable imaging doses. 


\section{Appendix 1}

\section{Data acquired in measurements of the detector sensitivity}

\begin{tabular}{|c|c|c|c|c|c|}
\hline Measurement & Signal (nC) & Measurement & Signal (nC) & Measurement & Signal $(\mathrm{nC})$ \\
\hline 1 & 8.448 & 50 & 8.337 & 99 & 8.398 \\
\hline 2 & 8.321 & 51 & 8.372 & 100 & 8.289 \\
\hline 3 & 8.281 & 52 & 8.282 & 101 & 8.296 \\
\hline 4 & 8.387 & 53 & 8.207 & 102 & 8.304 \\
\hline 5 & 8.286 & 54 & 8.260 & 103 & 8.321 \\
\hline 6 & 8.329 & 55 & 8.335 & 104 & 8.215 \\
\hline 7 & 8.310 & 56 & 8.306 & 105 & 8.297 \\
\hline 8 & 8.366 & 57 & 8.336 & 106 & 8.153 \\
\hline 9 & 8.385 & 58 & 8.271 & 107 & 8.336 \\
\hline 10 & 8.378 & 59 & 8.223 & 108 & 8.293 \\
\hline 11 & 8.293 & 60 & 8.273 & 109 & 8.335 \\
\hline 12 & 8.240 & 61 & 8.236 & 110 & 8.306 \\
\hline 13 & 8.296 & 62 & 8.309 & 111 & 8.292 \\
\hline 14 & 8.265 & 63 & 8.325 & 112 & 8.331 \\
\hline 15 & 8.385 & 64 & 8.325 & 113 & 8.265 \\
\hline 16 & 8.358 & 65 & 8.062 & 114 & 8.329 \\
\hline 17 & 8.339 & 66 & 8.380 & 115 & 8.196 \\
\hline 18 & 8.295 & 67 & 8.437 & 116 & 8.356 \\
\hline 19 & 8.370 & 68 & 8.207 & 117 & 8.381 \\
\hline 20 & 8.337 & 69 & 8.335 & 118 & 8.187 \\
\hline 21 & 8.211 & 70 & 8.171 & 119 & 8.382 \\
\hline 22 & 8.328 & 71 & 8.328 & 120 & 8.256 \\
\hline 23 & 8.387 & 72 & 8.374 & 121 & 8.293 \\
\hline
\end{tabular}


continued from previous page

\begin{tabular}{cccccc}
\hline \hline Measurement & Signal $(\mathrm{nC})$ & Measurement & Signal $(\mathrm{nC})$ & Measurement & Signal (nC) \\
\hline 24 & 8.158 & 73 & 8.314 & 122 & 8.334 \\
25 & 8.429 & 74 & 8.194 & 123 & 8.253 \\
26 & 8.293 & 75 & 8.279 & 124 & 8.367 \\
27 & 8.401 & 76 & 8.226 & 125 & 8.185 \\
28 & 8.351 & 77 & 8.338 & 126 & 8.307 \\
29 & 8.318 & 78 & 8.355 & 127 & 8.354 \\
30 & 8.319 & 79 & 8.345 & 128 & 8.311 \\
31 & 8.222 & 80 & 8.358 & 129 & 8.373 \\
32 & 8.297 & 81 & 8.351 & 130 & 8.372 \\
33 & 8.431 & 82 & 8.239 & 131 & 8.390 \\
34 & 8.379 & 83 & 8.355 & 132 & 8.380 \\
35 & 8.410 & 84 & 8.243 & 133 & 8.252 \\
36 & 8.261 & 85 & 8.307 & 134 & 8.285 \\
37 & 8.375 & 86 & 8.219 & 135 & 8.212 \\
38 & 8.236 & 87 & 8.347 & 136 & 8.311 \\
39 & 8.263 & 88 & 8.255 & 137 & 8.325 \\
40 & 8.385 & 89 & 8.268 & 138 & 8.328 \\
41 & 8.322 & 90 & 8.218 & 139 & 8.217 \\
42 & 8.240 & 91 & 8.240 & 140 & 8.369 \\
43 & 8.317 & 92 & 8.374 & 141 & 8.340 \\
44 & 8.221 & 93 & 8.414 & 142 & 8.352 \\
45 & 8.339 & 94 & 8.331 & 143 & 8.354 \\
46 & 8.256 & 95 & 8.388 & 144 & 8.289 \\
47 & 8.279 & 96 & 8.371 & 145 & 8.188 \\
48 & 8.266 & 97 & 8.215 & 146 & 8.209 \\
49 & 8.262 & 98 & 8.394 & & \\
\hline \hline
\end{tabular}




\section{References}

[1] Canadian Cancer Statistics Advisory Committee. Canadian cancer statistics 2017. Toronto, ON: Canadian Cancer Society; 2017. Available at: cancer.ca/Canadian-Cancer-Statistics-2017-EN.pdf [2018, May 29].

[2] J Sillanpaa, J Chang, G Mageras, E Yorke, F De Arruda, KE Rosenzweig, P Munro, E Seppi, J Pavkovich, and H Amols. Low-dose megavoltage cone-beam computed tomography for lung tumors using a high-efficiency image receptor. MEDICAL PHYSICS, 33(9):3489-3497, SEP 2006.

[3] AL Boyer, EB Butler, TA DiPetrillo, MJ Engler, B Fraass, W Grant, CC Ling, DA Low, TR Mackie, R Mohan, JA Purdy, M Roach, JG Rosenman, LJ Verhey, JW Wong, RL Cumberlin, H Stone, JR Palta, and Intensity Modulated Radiation Ther. Intensity-modulated radiotherapy: Current status and issues of interest. INTERNATIONAL JOURNAL OF RADIATION ONCOLOGY BIOLOGY PHYSICS, 51(4):880-914, NOV 152001.

[4] A Teymurazyan, JA Rowlands, and G Pang. Monte carlo simulation of a quantum noise limited cerenkov detector based on air-spaced light guiding taper for megavoltage x-ray imaging. MEDICAL PHYSICS, 41(4), APR 2014.

[5] Y El-Mohri, LE Antonuk, Q Zhao, RB Choroszucha, H Jiang, and L Liu. Low-dose megavoltage cone-beam ct imaging using thick, segmented scintillators. PHYSICS IN MEDICINE AND BIOLOGY, 56(6):1509-1527, 2011.

[6] FF Yin, HQ Guan, and WK Lu. A technique for on-board CT reconstruction using both kilovoltage and megavoltage beam projections for $3 \mathrm{D}$ treatment verification. MEDICAL PHYSICS, 32(9):2819-2826, 2005.

[7] J Pouliot, A Bani-Hashemi, J Chen, M Svatos, F Ghelmansarai, M Mitschke, M Aubin, P Xia, O Morin, K Bucci, M Roach, P Hernandez, ZR Zheng, D Hristov, and L Verhey. 
Low-dose megavoltage cone-beam CT for radiation therapy. INTERNATIONAL JOURNAL OF RADIATION ONCOLOGY BIOLOGY PHYSICS, 61(2):552-560, 2005.

[8] KM Langen, SL Meeks, DO Poole, TH Wagner, TR Willoughby, PA Kupelian, KJ Ruchala, J Haimerl, and GH Olivera. The use of megavoltage CT (MVCT) images for dose recomputations. PHYSICS IN MEDICINE AND BIOLOGY, 50(18):4259-4276, 2005.

[9] O Morin, J Chen, M Aubin, S Bose, A Gillis, M Bocci, and J Pouliot. Dose calculation using megavoltage cone beam CT imaging. INTERNATIONAL JOURNAL OF RADIATION ONCOLOGY BIOLOGY PHYSICS, 63(2, 1):S62-S63, 2005. 47th Annual Meeting of the American-Society-for-Therapeutic-Radiology-and-Oncology, Denver, CO, OCT 16-20, 2005 .

[10] Daniel Morf. Detector construction. In Ross I. Berbeco, editor, Beam's Eye View Imaging in Radiation Oncology, chapter 2. CRC Press, Boca Raton London New York, 2018.

[11] LE Antonuk. Electronic portal imaging devices: a review and historical perspective of contemporary technologies and research. PHYSICS IN MEDICINE AND BIOLOGY, 47(6):R31-R65, 2002.

[12] Ervin B. Podgoršak. Radiation Physics for Medical Physicists. Springer, Heidelberg Dordrecht London New York, 2 edition, 2010.

[13] Faiz M. Khan and John P. Gibbons. Khan's The Physics of Radiation Therapy. Lippincott Williams \& Wilkins, Philadelphia, 5 edition, 2014.

[14] JH Hubbell. Photon Cross Sections, Attenuation Coefficients, and Energy Absorption Coefficients From $10 \mathrm{keV}$ to $100 \mathrm{GeV}$. National Bureau of Standards, Washington, D.C., 1969.

[15] MG Herman, JM Balter, DA Jaffray, KP McGee, P Munro, S Shalev, M van Herk, and JW Wong. Clinical use of electronic portal imaging: Report of aapm radiation therapy committee task group 58. MEDICAL PHYSICS, 28(5):712-737, 2001.

[16] Ian A. Cunningham. Applied linear-system theory. In Jacob Beutel, Harold L. Kundel, and Richard L. van Metter, editors, Handbook of Medical Imaging Volum 1. Physics and Psychophysics, chapter 2. SPIE Press, Bellingham, Washington USA, 2000.

[17] James T. Dobbins III. Image quality metrics for digital systems. In Jacob Beutel, Harold L. Kundel, and Richard L. van Metter, editors, Handbook of Medical Imaging Volum 1. Physics and Psychophysics, chapter 3. SPIE Press, Bellingham, Washington USA, 2000. 
[18] C Kausch, B Schreiber, F Kreuder, R Schmidt, and O Dossel. Monte Carlo simulations of the imaging performance of metal plate/phosphor screens used in radiotherapy. MEDICAL PHYSICS, 26(10):2113-2124, 1999.

[19] BA Groh, JH Siewerdsen, DG Drake, JW Wong, and DA Jaffray. A performance comparison of flat-panel imager-based $\mathrm{MV}$ and $\mathrm{kV}$ cone-beam CT. MEDICAL PHYSICS, 29(6):967-975, 2002.

[20] A Sawant, LE Antonuk, Y El-Mohri, QH Zhao, YX Li, Z Su, Y Wang, J Yamamoto, H Du, I Cunningham, M Klugerman, and K Shah. Segmented crystalline scintillators: An initial investigation of high quantum efficiency detectors for megavoltage x-ray imaging. MEDICAL PHYSICS, 32(10):3067-3083, 2005.

[21] EJ Morton, W Swindell, DG Lewis, and PM Evans. A linear-array, scintillation crystal photodiode detector for megavoltage imaging. MEDICAL PHYSICS, 18(4):681-691, 1991.

[22] DG Lewis, W Swindell, EJ Morton, PM Evans, and ZR Xiao. A megavoltage CT scanner for radiotherapy verification. PHYSICS IN MEDICINE AND BIOLOGY, 37(10):19851999, 1992.

[23] MA Mosleh-Shirazi, PM Evans, W Swindell, JRN Symonds-Tayler, S Webb, and M Partridge. Rapid portal imaging with a high-efficiency, large field-of-view detector. $M E D I C A L$ PHYSICS, 25(12):2333-2346, 1998.

[24] MA Mosleh-Shirazi, W Swindell, and PM Evans. Optimization of the scintillation detector in a combined 3D megavoltage CT scanner and portal imager. MEDICAL PHYSICS, 25(10):1880-1890, 1998.

[25] EJ Seppi, P Munro, SW Johnsen, EG Shapiro, C Tognina, D Jones, JM Pavkovich, C Webb, I Mollov, LD Partain, and RE Colbeth. Megavoltage cone-beam computed tomography using a high-efficiency image receptor. INTERNATIONAL JOURNAL OF RADIATION ONCOLOGY BIOLOGY PHYSICS, 55(3):793-803, 2003.

[26] G Pang and JA Rowlands. Development of high quantum efficiency, flat panel, thick detectors for megavoltage x-ray imaging: A novel direct-conversion design and its feasibility. MEDICAL PHYSICS, 31(11):3004-3016, 2004.

[27] A Sawant, LE Antonuk, Y El-Mohri, QH Zhao, Y Wang, YX Li, H Du, and L Perna. Segmented crystalline scintillators: Empirical and theoretical investigation of a high quantum efficiency EPID based on an initial engineering prototype $\mathrm{CsI}(\mathrm{TI})$ detector. MEDICAL PHYSICS, 33(4):1053-1066, 2006. 
[28] TT Monajemi, BG Fallone, and S Rathee. Thick, segmented CdWO4-photodiode detector for cone beam megavoltage CT: A Monte Carlo study of system design parameters. MEDICAL PHYSICS, 33(12):4567-4577, 2006.

[29] TT Monajemi, D Tu, BG Fallone, and S Rathee. A bench-top megavoltage fan-beam CT using CdWO4-photodiode detectors. II. Image performance evaluation. MEDICAL PHYSICS, 33(4):1090-1100, 2006.

[30] Y Wang, LE Antonuk, Q Zhao, Y El-Mohri, and L Perna. High-DQE EPIDs based on thick, segmented BGO and CsI:Tl scintillators: Performance evaluation at extremely low dose. MEDICAL PHYSICS, 36(12):5707-5718, 2009.

[31] Y El-Mohri, LE Antonuk, Q Zhao, RB Choroszucha, H Jiang, and L Liu. Low-dose megavoltage cone-beam CT imaging using thick, segmented scintillators. PHYSICS IN MEDICINE AND BIOLOGY, 56(6):1509-1527, 2011.

[32] J Star-Lack, D Shedlock, D Swahn, D Humber, A Wang, H Hirsh, G Zentai, D Sawkey, I Kruger, M Sun, E Abel, G Virshup, M Shin, and R Fahrig. A piecewise-focused high DQE detector for MV imaging. MEDICAL PHYSICS, 42(9):5084-5099, 2015.

[33] Y Wang, LE Antonuk, Y El-Mohri, and Q Zhao. A Monte Carlo investigation of Swank noise for thick, segmented, crystalline scintillators for radiotherapy imaging. MEDICAL PHYSICS, 36(7):3227-3238, 2009.

[34] EK Breitbach, JS Maltz, B Gangadharan, A Bani-Hashemi, CM Anderson, SK Bhatia, J Stiles, DS Edwards, and RT Flynn. Image quality improvement in megavoltage cone beam CT using an imaging beam line and a sintered pixelated array system. MEDICAL PHYSICS, 38(11):5969-5979, 2011.

[35] S Rathee, D Tu, TT Monajemi, DW Rickey, and BG Fallone. A bench-top megavoltage fan-beam CT using CdWO4-photodiode detectors. I. System description and detector characterization. MEDICAL PHYSICS, 33(4):1078-1089, 2006.

[36] PF Kirvan, TT Monajemi, BG Fallone, and S Rathee. Performance characterization of a MVCT scanner using multislice thick, segmented cadmium tungstate-photodiode detectors. MEDICAL PHYSICS, 37(1):249-257, 2010.

[37] CWE van Eijk. Inorganic scintillators in medical imaging. PHYSICS IN MEDICINE AND BIOLOGY, 47(8):R85-R106, 2002. 
[38] J Rottmann, D Morf, R Fueglistaller, G Zentai, J Star-Lack, and R Berbeco. A novel EPID design for enhanced contrast and detective quantum efficiency. PHYSICS IN MEDICINE AND BIOLOGY, 61(17):6297-6306, 2016.

[39] H Chen, J Rottmann, SSF Yip, D Morf, R Fuglistaller, J Star-Lack, G Zentai, and $\mathrm{R}$ Berbeco. Super-resolution imaging in a multiple layer EPID. BIOMEDICAL PHYSICS \& ENGINEERING EXPRESS, 3(2), 2017.

[40] M Myronakis, J Star-Lack, P Baturin, J Rottmann, D Morf, A Wang, Y-H Hu, D Shedlock, and RI Berbeco. A novel multilayer MV imager computational model for component optimization. MEDICAL PHYSICS, 44(8):4213-4222, 2017.

[41] Y-H Hu, J Rottmann, R Fueglistaller, M Myronakis, A Wang, P Huber, D Shedlock, D Morf, P Baturin, J Star-Lack, and R Berbeco. Leveraging multi-layer imager detector design to improve low-dose performance for megavoltage cone-beam computed tomography. PHYSICS IN MEDICINE AND BIOLOGY, 63(3), 2018.

[42] G Pang and JA Rowlands. Development of high quantum efficiency flat panel detectors for portal imaging: Intrinsic spatial resolution. MEDICAL PHYSICS, 29(10):2274-2285, 2002 .

[43] X Mei and G Pang. Development of high quantum efficiency, flat panel, thick detectors for megavoltage x-ray imaging: An experimental study of a single-pixel prototype. MEDICAL PHYSICS, 32(11):3379-3388, 2005.

[44] X Mei, JA Rowlands, and G Pang. Electronic portal imaging based on Cerenkov radiation: A new approach and its feasibility. MEDICAL PHYSICS, 33(11):4258-4270, 2006.

[45] IC Silva and G Pang. Electronic portal imaging using Cherenkov radiation. In Hsieh, J and Samei, E, editor, MEDICAL IMAGING 2008: PHYSICS OF MEDICAL IMAGING, PTS 1-3, volume 6913 of Proceedings of SPIE. SPIE; Amer Assoc Phys Med; Amer Physiol Soc; Comp Assisted Radiol \& Surg; Soc Imaging Sci \& Technol; Med Image Percept Soc; Radiol Soc N Amer; Soc Imaging Informat Med; Soc Mol Imaging; DICOM Standards Comm, 2008. Medical Imaging 2008 Conference, San Diego, CA, FEB 17-19, 2008.

[46] A Teymurazyan and G Pang. An inherent anti-scatter detector for megavoltage x-ray imaging. PHYSICS IN MEDICINE AND BIOLOGY, 58(5):1479-1493, 2013.

[47] A Teymurazyan and G Pang. Monte Carlo simulation of a novel water-equivalent electronic portal imaging device using plastic scintillating fibers. MEDICAL PHYSICS, 39(3):1518$1529,2012$. 
[48] A Teymurazyan and G Pang. Megavoltage X-Ray Imaging Based on Cerenkov Effect: A New Application of Optical Fibres to Radiation Therapy. International Journal of Optics, 2012:724024, 2012.

[49] A Teymurazyan, JA Rowlands, and G Pang. Monte Carlo simulation of a quantum noise limited Cerenkov detector based on air-spaced light guiding taper for megavoltage x-ray imaging. MEDICAL PHYSICS, 41(4), 2014.

[50] Y Tian and G Pang. Experimental study of a single-pixel prototype anti-scatter detector for megavoltage x-ray imaging. RADIATION PHYSICS AND CHEMISTRY, 119:9-13, 2016.

[51] P Munro and DC Bouius. X-ray quantum limited portal imaging using amorphous silicon flat-panel arrays. MEDICAL PHYSICS, 25(5):689-702, 1998.

[52] M Rabbani, R Shaw, and R Vanmetter. Detective quantum efficiency of imaging-systems with amplifying and scattering mechanisms. JOURNAL OF THE OPTICAL SOCIETY OF AMERICA A-OPTICS IMAGE SCIENCE AND VISION, 4(5):895-901, 1987.

[53] JH Hubbell and SM Seltzer. NIST X-Ray Mass Attenuation Coefficients (ver. 1.4), [Online]. Available: https://www.nist.gov/pml/x-ray-mass-attenuation-coefficients [2018, May 28]. National Institute of Standards and Technology, Gaithersburg, MD., 2018.

[54] AL Boyer, L Antonuk, A Fenster, M van Herk, H Meertens, P Munro, LE Reinstein, and J Wong. A review of electronic protal imaging devices (EPIDS). MEDICAL PHYSICS, 19(1):1-16, 1992.

[55] W Zhao and JA Rowlands. X-ray imaging using amorphous selenium: Feasibility of a flat panel self-scanned detector for digital radiology. MEDICAL PHYSICS, 22(10):1595-1604, 1995.

[56] DWO Rogers. Fluence to dose equivalent conversion factors calculated with EGS3 for electrons from $100-\mathrm{keV}$ to $20 \mathrm{-GeV}$ and photons from $11-\mathrm{keV}$ to $20 \mathrm{-GeV}$. HEALTH PHYSICS, 46(4):891-914, 1984. 


\section{Acronyms}

AG air gap. 23, 24, 35, 37

BGO bismuth germanate. 13, 14

CBCT cone beam computed tomography. 2

CPID Cerenkov portal imaging detector. 15

CT computed tomography. 1, 3

CWO cadmium tungstate. 14

DQE detective quantum efficiency. v, $12,15,41$

EPID electronic portal imaging device. 2, 3, 13, 15, 35, 39, 41

GOS gadolinium oxysulfide. 14, 15

IGRT image-guided radiotherapy. 2, 3, 41

ISO isocenter. 14, 19, 20, 22, 23, 38, 39

Linac linear accelerator. 1, 2, 19, 24, 26, 28, 38, 39

LSF line spread function. 11, 20, 22, 23, 26, 34, 39

MRI magnetic resonance imaging. 1,3

MTF modulation transfer function. 12, 15, 20, 23, 34

MU monitor unit. 19, 24, 26, 39 
NPS noise power spectrum. 12, 13,41

PET positron emission tomography. 1

PMT photomultiplier tube. $\mathrm{x}, 17,19,23,26,29,31,39,40$

PSF point spread function. 11, 12

QE quantum efficiency. v, 12, 15, 20, 25, 27, 34, 38, 39, 41, 42

rms root-mean-square. $26,28,38$

SAD source to axis distance. 19

SNR signal to noise ratio. v, 11, 12, 15, 41

SPR scatter to primary ratio. 23, 25, 35, 37, 39 